\title{
Experimental and theoretical study of the electronic structure of $\mathrm{HgO}$ and $\mathrm{Tl}_{2} \mathrm{O}_{3}$
}

\author{
Per-Anders Glans, Timothy Learmonth, and Kevin E. Smith \\ Department of Physics, Boston University, 590 Commonwealth Avenue, Boston, Massachusetts 02215, USA \\ Jinghua Guo \\ Advanced Light Source, Lawrence Berkeley National Laboratory, Berkeley, California 94720, USA
}

Aron Walsh and Graeme W. Watson

Department of Chemistry, University of Dublin, Trinity College, Dublin 2, Ireland

Fabio Terzi* and Russell G. Egdell ${ }^{\dagger}$

Department of Chemistry, Inorganic Chemistry Laboratory, South Parks Road, Oxford OX1 3QR, United Kingdom

(Received 26 February 2005; published 22 June 2005)

\begin{abstract}
The electronic structures of $\mathrm{HgO}$ and $\mathrm{Tl}_{2} \mathrm{O}_{3}$ have been investigated by valence and core-level x-ray photoemission, $\mathrm{x}$-ray absorption, and x-ray emission spectroscopies. Valence-band photoemission under $\mathrm{Al} K \alpha$ excitation is dominated by the metal $5 d$ partial density of states and thus provides a sensitive probe of shallow core mixing into the $\mathrm{O} 2 p$ valence-band states. Conversely $\mathrm{O} K$ shell emission is determined by the $\mathrm{O} 2 p$ partial density of states and therefore allows the extent of corresponding mixing of $\mathrm{O} 2 p$ character into the shallow core states to be measured. The experimental work is supported by band-structure calculations carried out within the framework of density-functional theory. It is shown that the bonding in $\mathrm{HgO}$ involves significant mixing between $\mathrm{O} 2 p$ states and both $\mathrm{Hg} 6 s$ and shallow core $5 d$ states: the calculated $\mathrm{O} 2 p$ partial density of states mirrors the $\mathrm{O} K$ shell emission spectrum and reveals significant $\mathrm{O} 2 p$ character within the shallow core $\mathrm{Hg} 5 d$ states. There is, however, little direct on-site mixing between the $\mathrm{Hg} 6 s$ and $5 d$ states. $\mathrm{In}_{2} \mathrm{Tl}_{2} \mathrm{O}_{3}$, the hybridization of the deeper metal $5 d$ states with $\mathrm{O} 2 p$ states is much less pronounced than in $\mathrm{HgO}$. Moreover, the states at the bottom of what is conventionally regarded as the $\mathrm{O} 2 p$ valence band are found in fact to have very strong $\mathrm{Tl} 6 \mathrm{~s}$ atomic character. The photoemission spectrum of $\mathrm{Tl}_{2} \mathrm{O}_{3}$ shows a well-defined metallic Fermi edge: the shape of the structure around the photoemission onset suggests that the metallic nature of $\mathrm{Tl}_{2} \mathrm{O}_{3}$ arises from an occupation of states above the main valence-band edge, probably arising from oxygen vacancy defects. The conduction electrons of $\mathrm{Tl}_{2} \mathrm{O}_{3}$ are strongly perturbed by ionization of $\mathrm{Tl}$ core levels, giving rise to distinctive plasmon satellites in core $\mathrm{x}$-ray photoemission spectroscopy.
\end{abstract}

DOI: 10.1103/PhysRevB.71.235109

PACS number(s): 78.70.En, 79.60.-i

\section{INTRODUCTION}

The two families of cuprate superconductors with the highest known $T_{c}$ values are complex layered materials incorporating $\mathrm{Hg}$ and $\mathrm{Tl}$, respectively. ${ }^{1-6}$ The heavy posttransition elements are generally presumed to be "spectators" of the electronic structure, that is, they do not contribute directly to the electronic states close to the Fermi level. Instead, electronic "activity" is assumed to be confined to the $\mathrm{CuO}_{2}$ planes common to all cuprate superconductors and the heavy-element layers are treated simply as charge reservoirs. ${ }^{7,8}$ Although the electronic structure of complex cuprate phases has been intensively studied in the past few years, ${ }^{9}$ surprisingly little attention has been devoted to the simple binary oxides $\mathrm{HgO}$ and $\mathrm{Tl}_{2} \mathrm{O}_{3}$. In particular, there is a paucity of photoemission or other high-energy spectroscopic data for these materials. The structure of the valence band is poorly defined in previously published He I and X-ray photoemission spectra for $\mathrm{HgO}$ (Refs. 10 and 11), and we are unaware of any valence-band photoemission data at all for $\mathrm{Tl}_{2} \mathrm{O}_{3}$. In the present paper, we present core- and valencelevel X-ray photoemission spectra (XPS) of $\mathrm{HgO}$ and $\mathrm{Tl}_{2} \mathrm{O}_{3}$ and compare the valence-band data with $\mathrm{X}$-ray emission spectra (XES). The two sets of experimental spectra are com- pared in turn with total and partial density of states profiles derived from band-structure calculations carried out within the framework of density-functional theory. We also explore resonance effects in $\mathrm{x}$-ray emission and consider the relationship between X-ray absorption (XAS) and emission spectra.

$\mathrm{HgO}$ has two important polymorphs: a yellow hexagonal phase stable above $220^{\circ} \mathrm{C}$ and a low-temperature red orthorhombic phase. ${ }^{12}$ The band gap of orthorhombic $\mathrm{HgO}$ is $1.90 \mathrm{eV} .^{13,14}$ Both phases are based on zigzag -Hg-O-Hg-O- chains with coordination about $\mathrm{Hg}$ which is essentially linear and an $\mathrm{Hg}-\mathrm{O}-\mathrm{Hg}$ bond angle close to the tetrahedral value. The chains in the hexagonal phase have a spiral architecture, but in the more important orthorhombic phase the chains are planar, as shown in Fig. 1. In contrast to these structures, the group congener $\mathrm{ZnO}$ adopts the wurtzite structure with tetrahedral metal coordination, while $\mathrm{CdO}$ crystallizes in the rocksalt structure with regular octahedral coordination about $\mathrm{Cd}$. The conventional textbook explanation ${ }^{15,16}$ of the linear stereochemistry found in $\mathrm{HgO}$ and other mercury compounds is that this coordination environment promotes intra-atomic mixing between the filled shallow core $\mathrm{Hg} 5 d_{z}^{2}$ states and the nominally empty $\mathrm{Hg} 6 \mathrm{~s}$ states, thus lowering the internal electronic energy of the $\mathrm{Hg}$ II ions through a second-order Jahn-Teller effect. The on-site 


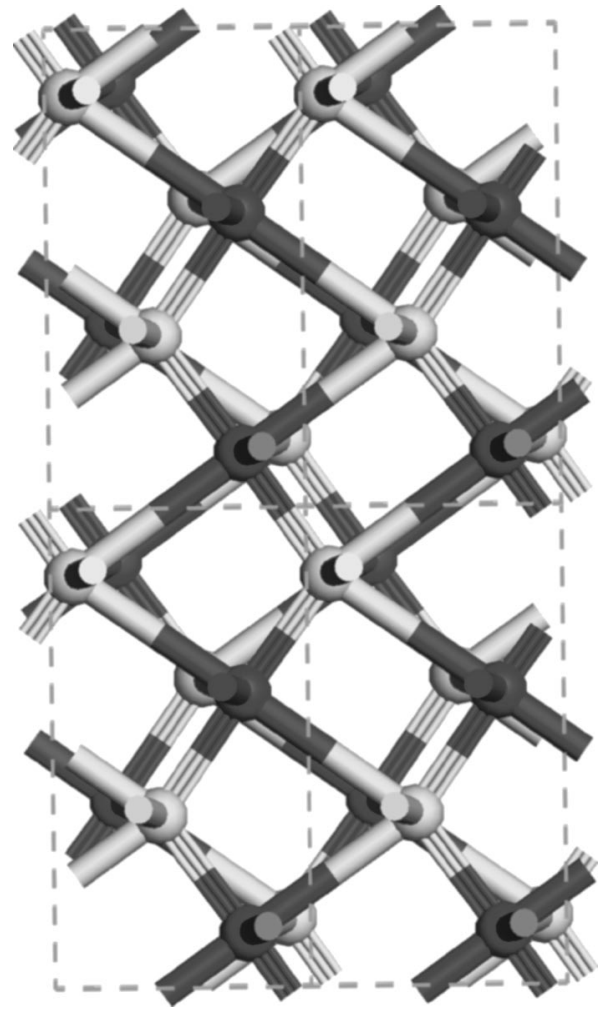

FIG. 1. Four unit cells of orthorhombic $\mathrm{HgO}$. Each cell contains four formula units. $\mathrm{Hg}$ atoms are dark gray, $\mathrm{O}$ atoms light gray. The planar zigzag $\mathrm{O}-\mathrm{Hg}-\mathrm{O}$ chains discussed in the text are highlighted by fluting along the bonds.

hybridization with the shallow core level should be more pronounced in $\mathrm{Hg}$ compounds than in $\mathrm{Zn}$ or $\mathrm{Cd}$ compounds because the $\mathrm{Hg} 5 d$ binding energy is lower than $\mathrm{Cd} 4 d$ and $\mathrm{Zn} 3 d$ binding energies, while the $\mathrm{Hg} 6 s$ level lies at higher binding energy than the $\mathrm{Cd} 5 s$ and $\mathrm{Zn} 4 s$ levels. ${ }^{17}$ Thus the difference in energy between $6 s$ and $5 d$ levels in $\mathrm{Hg}$ is very much lower than corresponding separations in $\mathrm{Zn}$ and $\mathrm{Cd}$, leading to the hybridization-driven propensity for linear coordination in $\mathrm{HgO}$. The atomic binding energies in $\mathrm{Hg}$ are in turn strongly influenced by relativistic effects which stabilize the $\mathrm{Hg} 6 s$ levels and at the same time lower the binding energy of the shallow core $\mathrm{Hg} 5 d$ levels. ${ }^{18}$ In a previous communication, ${ }^{19}$ we have used nonresonant XES to demonstrate more pronounced involvement of the shallow core $d$ levels in the bonding in $\mathrm{HgO}$ than in $\mathrm{ZnO}$ or $\mathrm{CdO}^{20}$

The second oxide of interest here, namely $\mathrm{Tl}_{2} \mathrm{O}_{3}$, is a dark brown compound that adopts the lanthanoid-C structure (alternatively known as bixbyite) under ambient conditions. ${ }^{12,21}$ This is derived from a $(2 \times 2 \times 2)$ superstructure of fluorite with one-quarter of the anion sites vacant (Fig. 2). The resulting sixfold-coordinated $\mathrm{Tl}$ atoms are of two types. For one-quarter of the $\mathrm{Tl}$ ions, two $\mathrm{O}$ ions are missing from the body diagonal of the cube defining the metal coordination in fluorite, while for the remaining $\mathrm{Tl}$ ions the two missing $\mathrm{O}$ ions are at the ends of a face diagonal. Thus in the oxide there is no tendency for $\mathrm{Tl}$ to adopt the linear coordination found in $\mathrm{HgO}$, even though $\mathrm{Hg}^{2+}$ and $\mathrm{Tl}^{3+}$ are formally isoelectronic.

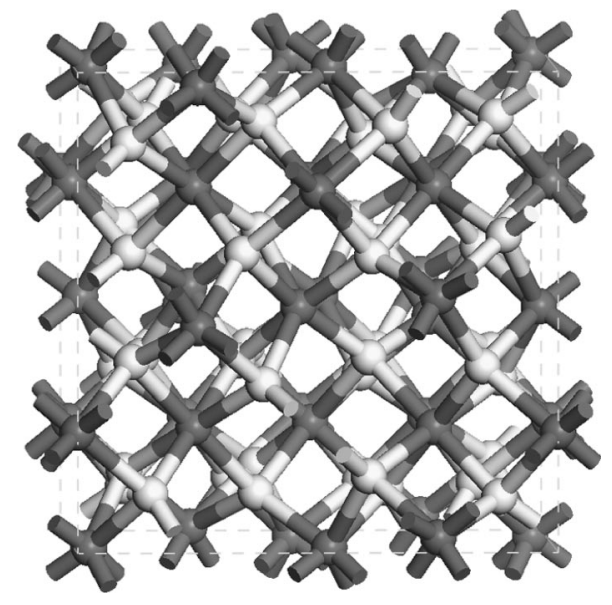

FIG. 2. Unit cell of cubic $\mathrm{Tl}_{2} \mathrm{O}_{3}$. There are 16 formula units per cell and therefore 80 atoms in the unit cell. Tl atoms are dark gray, $\mathrm{O}$ atoms light gray.

It has been known for some time that $\mathrm{Tl}_{2} \mathrm{O}_{3}$ is a metallic conductor, ${ }^{22-27}$ but there is no consensus as to whether this is an intrinsic property of the stoichiometric phase $\mathrm{Tl}_{2} \mathrm{O}_{3}$ or arises from oxygen deficiency in $\mathrm{Tl}_{2} \mathrm{O}_{3-x}$. The latter interpretation is tacitly favored by a value of $1.60 \mathrm{eV}$ quoted in the recent literature for the "band gap" in $\mathrm{Tl}_{2} \mathrm{O}_{3}{ }^{9}, 10$ And it is certainly the case that although samples of $\mathrm{Tl}_{2} \mathrm{O}_{3}$ are invariably metallic, the carrier concentration varies strongly with oxygen partial pressure in the way that would be expected if the carriers arose from ionized oxygen vacancies. ${ }^{24,26,27} \mathrm{On}$ the other hand, a recent band-structure calculation suggests that the neighboring oxide $\mathrm{PbO}_{2}$ is intrinsically metallic ${ }^{28}$ due to overlap of the bottom of the $\mathrm{Pb} 6 s$ conduction band with the top of the $\mathrm{O} 2 p$ valence band and this situation must be regarded as a possibility for $\mathrm{Tl}_{2} \mathrm{O}_{3}$. Until now the large size of the unit cell has prevented the study of the electronic structure of $\mathrm{Tl}_{2} \mathrm{O}_{3}$ by first-principles electronic bandstructure calculations. The electronic properties of the isostructural compound $\mathrm{In}_{2} \mathrm{O}_{3}$ are rather better understood. This oxide is intrinsically an insulator with a direct band gap of $3.75 \mathrm{eV}$ (Ref. 29) and a smaller indirect gap of $2.6 \mathrm{eV} .{ }^{30}$ As with $\mathrm{CdO},{ }^{31,32}$ the indirect gap appears to arise from strong mixing between shallow core $d$ states and $\mathrm{O} 2 p$ states away from the zone center. $\operatorname{In}_{2} \mathrm{O}_{3}$ is easily doped $n$-type either by oxygen deficiency ${ }^{29}$ or by substitution of $\mathrm{Sn}$ on In sites ${ }^{33}$ to give a highly degenerate transparent conducting oxide.

\section{EXPERIMENT}

High-resolution $\mathrm{x}$-ray photoemission spectra were measured in a Scienta ESCA 300 spectrometer at the National Centre for Electron Spectroscopy and Surface Analysis (NCESS) at Daresbury Laboratory (UK). This incorporates a rotating anode $\mathrm{Al} K \alpha(h \nu=1486.6 \mathrm{eV}) \mathrm{x}$-ray source, a sevencrystal x-ray monochromator, and a $300 \mathrm{~mm}$ mean radius spherical sector electron energy analyzer with a parallel electron detection system. The $\mathrm{x}$-ray source was run with $200 \mathrm{~mA}$ emission current and $14 \mathrm{kV}$ anode bias, while the analyzer operated at $150 \mathrm{eV}$ pass energy with $0.8 \mathrm{~mm}$ slits. 
Gaussian convolution of the analyzer resolution with a linewidth of $260 \mathrm{meV}$ for the $\mathrm{x}$-ray source gives an effective instrument resolution of $350 \mathrm{meV}$. Phase-pure orthorhombic $\mathrm{HgO}$ (Sigma-Aldrich 99.999\%) and cubic $\mathrm{Tl}_{2} \mathrm{O}_{3}$ (SigmaAldrich $99.99 \%$ ) were heated ex situ at $100^{\circ} \mathrm{C}$ for $24 \mathrm{~h}$ to minimize surface contamination by water or carbonates and were then pressed into In foil. In situ, the samples were subject to further gentle heating to $100^{\circ} \mathrm{C}$ to minimize the level of adventitious $\mathrm{C}$ contamination. The $\mathrm{C} 1 s$ to $\mathrm{O} 1 s$ intensity ratio was reduced to below 0.02 for both oxides. The spectra were also completely free of core-level structure associated with the In support (in particular the very strong In $3 d_{5 / 2}$ peak at $\approx 445 \mathrm{eV}$ binding energy). For metallic $\mathrm{Tl}_{2} \mathrm{O}_{3}$, binding energies are referenced to the midpoint of the Fermi edge observed in valence region photoemission. Sample charging was problematic for $\mathrm{HgO}$ and it was necessary to stabilize the surface charge with a flood gun delivering $4 \mathrm{eV}$ electrons. Binding energies for $\mathrm{HgO}$ are referenced to the weak residual $\mathrm{C} 1 s$ peak, which was taken to have a binding energy of $285.0 \mathrm{eV}$.

All x-ray absorption and emission spectra were measured at beamline 7.0.1 at the Advanced Light Source (ALS), Lawrence Berkeley National Laboratory, USA. This beamline is equipped with a spherical grating monochromator. ${ }^{34}$ Emission spectra were recorded using a Nordgren-type grazing-incidence spherical grating spectrometer. ${ }^{35}$ For the emission experiments, the beamline was set to have an energy resolution of $500 \mathrm{meV}$ at the $\mathrm{O} K$ edge and $50 \mathrm{meV}$ at the $\mathrm{Hg} N_{6,7}$ edges, respectively. The spectrometer was set to have a resolution of $500 \mathrm{meV}$ for $\mathrm{O} K$ and $72 \mathrm{meV}$ for $\mathrm{Hg}$ $N_{6,7}$ edges. Absorption spectra were measured in total electron yield (TEY) mode by monitoring the sample drain current. For the absorption measurements, the beamline resolution was set to $200 \mathrm{meV}$ for the $\mathrm{O} K$ edge and to $50 \mathrm{meV}$ for the $\mathrm{Hg} N_{6,7}$ edges. The absorption spectra were normalized to a reference current from a clean gold mesh positioned in the path of the photon beam.

\section{COMPUTATIONS}

Density-functional theory as embodied in the Vienna Abinitio Simulation Package (VASP) ${ }^{36,37}$ was used to calculate the electronic structure of $\mathrm{HgO}$ and $\mathrm{Tl}_{2} \mathrm{O}_{3}$. The calculations included explicit treatment of the spin-orbit splitting of the shallow core levels. The crystal wave functions were expanded in terms of a plane-wave basis set using periodic boundary conditions. The generalized gradient approximation (GGA) parametrization of Perdew, Burke, and Ernzerhof ${ }^{38}$ was used with the projector augmented wave method $^{39}$ employed to represent the valence-core interactions $(\mathrm{Hg}, \mathrm{Tl}:[\mathrm{Xe}], \mathrm{O}:[\mathrm{He}])$. These fixed core states were generated from all-electron scalar relativistic calculations. Calculations on a free $\mathrm{Hg}$ atom show a $1.83 \mathrm{eV}$ splitting of the $5 d$ states and a separation of $2.43 \mathrm{eV}$ between the $5 d_{5 / 2}$ and $6 \mathrm{~s}$ levels. These are in reasonable agreement with the DiracFock calculations, indicating that the spin-orbit approach in addition to the relativistic frozen-core electrons and their subsequent effect on the valence electrons give rise to a good representation of the relativistic effects in $\mathrm{Hg}$.
TABLE I. Structural data for $\mathrm{HgO}$ derived from DFT calculations.

\begin{tabular}{cccc}
\hline \hline & Experiment & Calculation & \% Error \\
\hline Energy $(\mathrm{eV} / \mathrm{Hg})$ & & -23.31 & \\
Volume $\left(\AA^{3}\right)$ & 128.51 & 140.89 & $+9.86 \%$ \\
$a(\AA)$ & 6.61 & 6.74 & $+2.0 \%$ \\
$b(\AA)$ & 5.52 & 5.68 & $+2.8 \%$ \\
$c(\AA)$ & 3.52 & 3.68 & $+4.4 \%$ \\
Bond lengths $(\AA)$ & $1 \times 2.02,1 \times 2.05$ & $1 \times 2.07,1 \times 2.10$ \\
& $2 \times 2.82$ & $2 \times 2.91$ & \\
& $1 \times 2.83,1 \times 2.89$ & $1 \times 2.93,1 \times 2.99$ \\
\hline
\end{tabular}

The calculations were checked for convergence with respect to both plane-wave cutoff $(500 \mathrm{eV}$ was used for $\mathrm{HgO}$ and $\left.\mathrm{Tl}_{2} \mathrm{O}_{3}\right)$ and $k$-point sampling $(4 \times 4 \times 6$ and $2 \times 2 \times 2$ grids were used for $\mathrm{HgO}$ and $\mathrm{Tl}_{2} \mathrm{O}_{3}$, respectively). Optimization at a series of volumes was performed, allowing the atomic positions, the lattice vectors, and angles to relax within constrained total volume. The resulting energy volume curve was fitted to the Murnaghan equation of state to obtain the equilibrium cell volume. This approach was taken to avoid the problem of the Pulay stress and changes in basis set that occur in plane-wave calculations on volume changes.

Structural optimization of $\mathrm{HgO}$ resulted in the cell parameters shown in Table I. The percentage deviations from the experimental values are seen to be quite small. Figure 3 shows the coordination environment around $\mathrm{Hg}$ in the equilibrium unit cell. The nearest-neighbor $\mathrm{Hg}-\mathrm{O}$ interatomic distance is calculated to be $2.07 \AA$, which is only $2.0 \%$ greater than the experimental value of $2.03 \AA$ The next nearest coordinated oxygen is found at $2.10 \AA$, with two further $\mathrm{O}$ atoms at $2.91 \AA$ and the remaining two at distances of $2.93 \AA$ and $2.99 \AA$. These are in good agreement with ex-

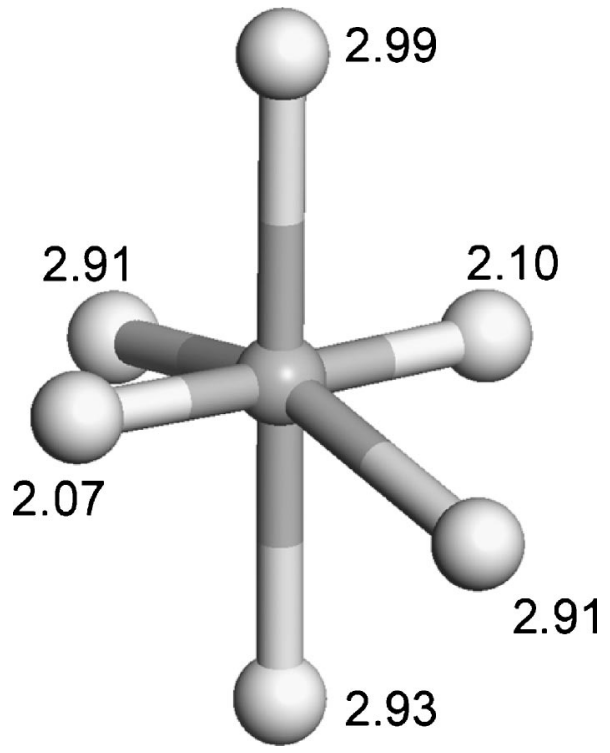

FIG. 3. The Hg-O nearest-neighbor interatomic distances $(\AA)$ in the optimized $\mathrm{HgO}$ crystal structure resulting from the DFT calculations. 
TABLE II. Structural data for $\mathrm{Tl}_{2} \mathrm{O}_{3}$ derived from DFT calculations.

\begin{tabular}{cccc}
\hline \hline & Experiment & Calculation & \% Error \\
\hline Energy $(\mathrm{eV} / \mathrm{Tl})$ & & -9.97 & \\
Volume $\left(\AA^{3}\right)$ & 1167.58 & 1250.84 & $+7.1 \%$ \\
$a(\AA)$ & 10.53 & 10.78 & $+2.3 \%$ \\
$b(\AA)$ & 10.53 & 10.78 & $+2.3 \%$ \\
$c(\AA)$ & 10.53 & 10.78 & $+2.3 \%$ \\
Bond lengths & $6 \times 2.27$ & $6 \times 2.32$ & \\
Tl type I $(\AA)$ & & & \\
Bond lengths & $2 \times 2.20$ & $2 \times 2.25$ & \\
Tl type II $(\AA)$ & & & \\
& $2 \times 2.29$ & $2 \times 2.34$ & \\
\hline \hline
\end{tabular}

periment, as can be seen in Table I. Optimization of $\mathrm{Tl}_{2} \mathrm{O}_{3}$ yielded the cell parameters shown in Table II. The calculated lattice vectors are also in good agreement with experiment: the calculated cubic cell parameter is within $2.3 \%$ of the experimentally determined value. The coordination around the two types of $\mathrm{Tl}$ is shown in Fig. 4. The first type of $\mathrm{Tl}$ features six oxygen atoms around $\mathrm{Tl}$ all at a distance of $2.32 \AA$, as shown in Fig. 4(a). The second type of Tl site is less symmetric with three pairs of two oxygen atoms at $2.34 \AA$, $2.37 \AA$, and $2.45 \AA$, respectively [Fig. 4(b)].

The partial (ion and $l$ - and $m$-quantum number decomposed) electronic density of states (PEDOS) has been calculated for both $\mathrm{HgO}$ and $\mathrm{Tl}_{2} \mathrm{O}_{3}$. These were obtained by projecting the wave functions onto spherical harmonics centered on each atom with a radius of $1.60 \AA$ for both $\mathrm{Hg}$ and $\mathrm{Tl}$ and a radius of $1.55 \AA$ for $\mathrm{O}$ atoms. These radii were chosen because they give rise to reasonable space filling and the correct number of electrons, but the results (at least the qualitative aspects) are insensitive to a change of the radii.

\section{RESULTS AND DISCUSSION}

\section{A. The calculated densities of states}

The calculated total and partial densities of states for $\mathrm{HgO}$ are shown in Fig. 5. It is convenient to discuss the occupied states below the Fermi energy in terms of five peaks labeled $\mathrm{I}-\mathrm{V}$ in the figure. Inspection of the partial densities of states in the lower panel reveals that the three peaks at lowest binding energy (I, II, and III) have dominant $\mathrm{O} 2 p$ atomic character, whereas IV and V are of dominant $\mathrm{Hg} 5 d$ character. However, there is significant mixing between $\mathrm{O} 2 p$ and $\mathrm{Hg}$ $5 d$ states, so that the "Hg $5 d$ " states IV and V have substantial $\mathrm{O} 2 p$ atomic character while the "O $2 p$ " states I-III have equally significant $\mathrm{Hg} 5 d$ character: the admixture with $\mathrm{Hg}$ $5 d$ is particularly pronounced in the highest binding-energy peak III, which has about $20 \% \mathrm{Hg} 5 d$ character. At the same time, there is also pronounced mixing between $\mathrm{O} 2 p$ and the $\mathrm{Hg} 6 s$ and $6 p$ states, which are notionally empty in the ionic limit. Within the occupied states, the mixing with $\mathrm{Hg} 6 s$ is most pronounced in peak III, which has about $20 \% \mathrm{Hg} 6 \mathrm{~s}$
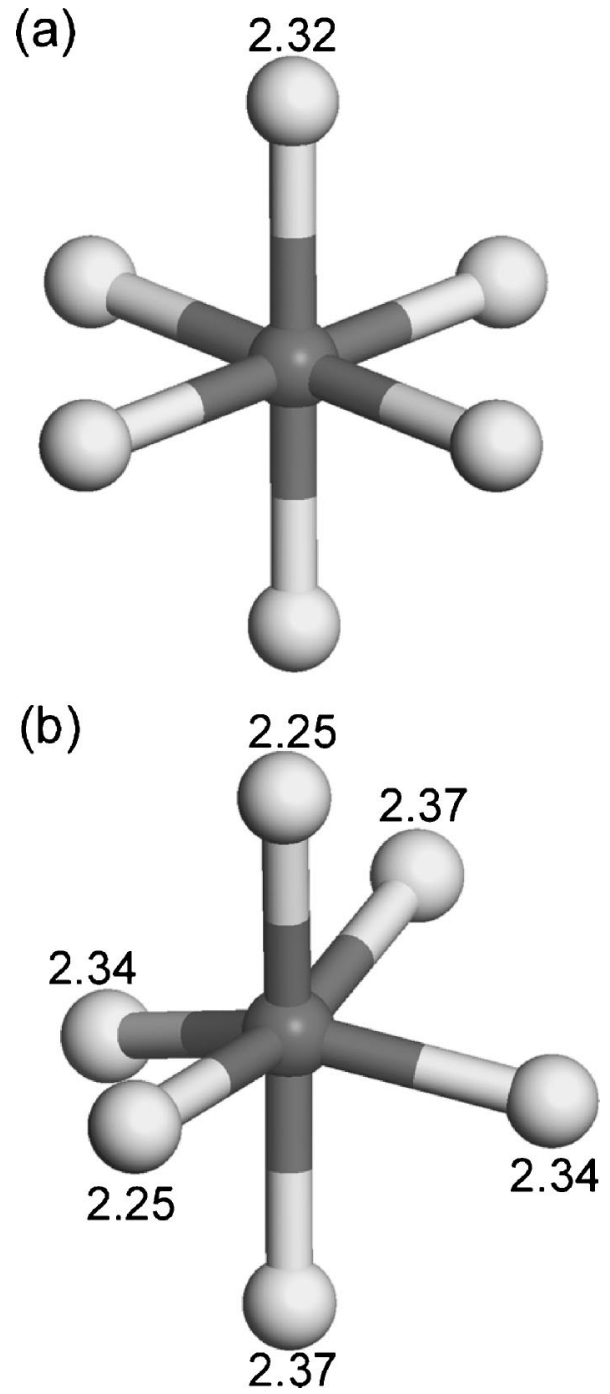

FIG. 4. The Tl-O nearest-neighbor interatomic distances $(\AA)$ for two types of $\mathrm{Tl}$ site in the optimized $\mathrm{Tl}_{2} \mathrm{O}_{3}$ crystal structure resulting from the DFT calculations.

character, with a more limited $6 s$ contribution to peaks I and II. At the same time, the most pronounced mixing with $\mathrm{Hg}$ $6 p$ states is found in band II. Correspondingly, the lowest conduction-band states are an essentially equal mix of $\mathrm{Hg} 6 \mathrm{~s}$ and $\mathrm{O} 2 p$ atomic character, and the most pronounced $\mathrm{Hg} 6 p$ character in the empty states is found between $4 \mathrm{eV}$ and $10 \mathrm{eV}$ above the Fermi level. Overall, it can be seen that the lowest "O $2 p$ " valence state III contains a substantial contribution from both $\mathrm{Hg} 6 s$ and $\mathrm{Hg} 5 d$ states. There is, however, little direct on-site mixing between $\mathrm{Hg} 6 s$ and $\mathrm{Hg} 5 d$ : empty conduction-band states have only limited $\mathrm{Hg} 5 d$ character and there is a correspondingly small contribution of $\mathrm{Hg} 6 \mathrm{~s}$ character to the states in bands IV and V. The dominant $\mathrm{Hg}$ $6 p$ contribution to the $\mathrm{O} 2 p$ valence band appears in the central band II.

It is necessary to consider structure within the $5 d$ region in further detail. The spin-orbit interaction splits the $5 d$ level into $5 d_{5 / 2}$ and $5 d_{3 / 2}$ levels. In the absence of further interactions, the $5 d$ density of states will consist of just two components with integrated areas in the ratio 6:4. However, co- 


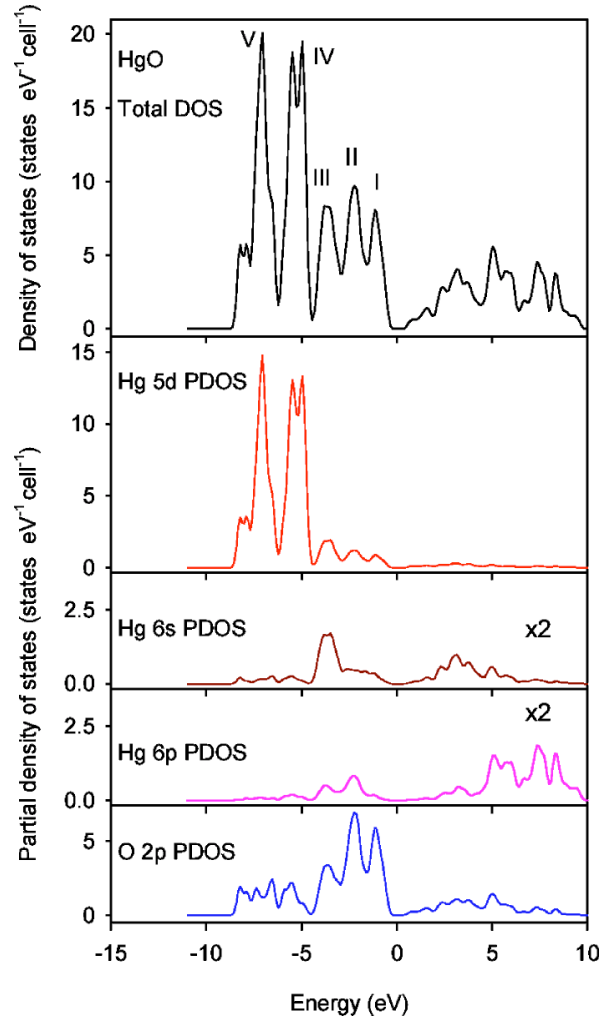

FIG. 5. The total and partial densities of states for orthorhombic $\mathrm{HgO}$. The $\mathrm{Hg} 6 s$ and $\mathrm{Hg} 6 p$ partial densities of states are presented on an energy scale expanded by a factor of 2 relative to the other data.

valent interactions with the $\mathrm{O} 2 p$ levels can further split the $5 d$ levels. Due to the fact that the two nearest-neighbor oxygen atoms are almost $1 \AA$ closer to the $\mathrm{Hg}$ than the remaining four oxygen atoms completing the coordination sphere, the splitting of the $5 d$ levels is basically that found in a linear (point group $D_{\infty \mathrm{h}}$ ) environment. In the nonrelativistic limit, a $d$ shell will split to give $\delta$, $\pi$, and $\sigma$ levels: the spin-orbit interaction further splits $\delta$ (which has $\lambda=2$ ) into $\delta_{5 / 2}$ and $\delta_{3 / 2}$ and $\pi(\lambda=1)$ into $\pi_{3 / 2}$ and $\pi_{1 / 2}$, leaving the nondegenerate $\sigma$ level $(\lambda=0)$ as $\sigma_{1 / 2}$. Starting from the relativistic limit, the perturbation due to the molecular field can alternatively be described in terms of splitting of the $5 d_{5 / 2}$ level into $\delta_{5 / 2}$, $\pi_{3 / 2}$, and $\sigma_{1 / 2}$ components, while $5 d_{3 / 2}$ splits to give $\delta_{3 / 2}$ and $\pi_{1 / 2}$. Since there are no O $2 p$ orbitals of $\delta$ symmetry and $\pi$ interactions are weaker than $\sigma$ interactions, the ordering of the levels derived from $5 d_{5 / 2}$ in order of increasing binding energy will be $\delta<\pi<\sigma$. Splittings within both the $5 d_{5 / 2}$ and $5 d_{3 / 2}$ levels are found in the calculated density of states and it emerges that the downward shift of the $\sigma_{1 / 2}$ level is so pronounced that this structure overlaps $5 d_{3 / 2}$ structure in band $\mathrm{V}$ rather than falling within the contour of band IV.

Turning next to $\mathrm{Tl}_{2} \mathrm{O}_{3}$, it is again convenient to discuss states below the Fermi level in terms of five bands labeled $\mathrm{I}-\mathrm{V}$ in Fig. 6. As with $\mathrm{HgO}$, IV and $\mathrm{V}$ are of dominant $5 d$ character and I-III correspond to what are conventionally regarded as the $\mathrm{O} 2 p$ valence-band states. The $5 d$ levels are more tightly bound in $\mathrm{Tl}_{2} \mathrm{O}_{3}$ than $\mathrm{HgO}$ and, not surprisingly, mixing between $\mathrm{Tl} 5 d$ and $\mathrm{O} 2 p$ levels is less pronounced

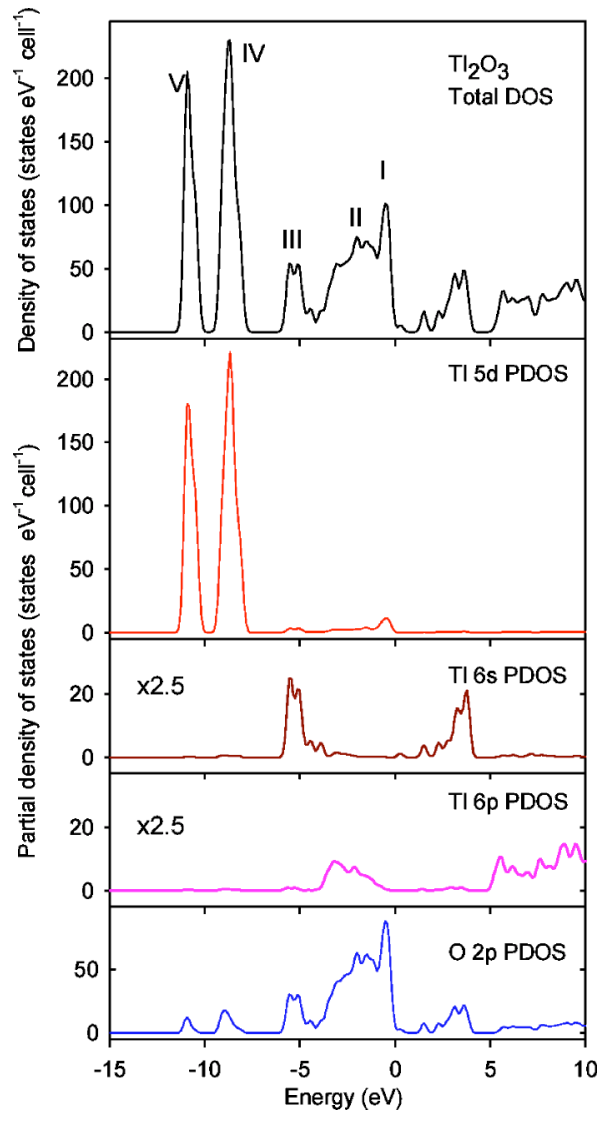

FIG. 6. The total and partial densities of states for cubic $\mathrm{Tl}_{2} \mathrm{O}_{3}$. The Tl $6 s$ and $\mathrm{Tl} 6 p$ partial densities of states are presented on an energy scale expanded by a factor of 2.5 relative to the other data.

than the corresponding mixing between $\mathrm{Hg} 5 d$ and $\mathrm{O} 2 p$ in $\mathrm{HgO}$. Moreover, there is no pronounced ligand field splitting of the $5 d$ levels. Thus the $5 d$ structure is essentially a simple spin-orbit doublet with around 5\% O $2 p$ character in the $5 d_{5 / 2}$ level and $3 \%$ O $2 p$ character in the more tightly bound $5 d_{3 / 2}$ level. Correspondingly, there is less $5 d$ character within the "O $2 p$ " bands I-III than is found in $\mathrm{HgO}$. Moreover, the most pronounced $\mathrm{Tl} 5 d$ character is found in the topmost $\mathrm{O}$ $2 p$ band I, rather than in III. At the same time, the extent of mixing between $\mathrm{O} 2 p$ and metal $6 s$ levels is greater than in $\mathrm{HgO}$. In particular, the most tightly bound "O $2 p$ " band III contains an almost equally balanced mix of $\mathrm{Tl} 6 s$ and $\mathrm{O} 2 p$ atomic character.

The final issue of interest in relation to $\mathrm{Tl}_{2} \mathrm{O}_{3}$ is that the density of states does not go to zero at the Fermi energy. Thus $\mathrm{Tl}_{2} \mathrm{O}_{3}$ emerges in the calculations as an intrinsically metallic material. The Fermi energy lies toward the top of the uppermost $\mathrm{O} 2 p$ band $\mathrm{I}$, but there is a further weak peak in the density of states just above the Fermi energy seen in the expanded plot of Fig. 7. The density of states declines to a very low value at yet higher energy (but without a true gap) before a further peak about $1.5 \mathrm{eV}$ above the Fermi energy. $(E, k)$ dispersion curves reveal that a single band with a minimum energy about midway along the $\Gamma-X$ direction disperses upward from just below the Fermi energy to an energy of about $2 \mathrm{eV}$ at the $\Gamma$ point. The metallic nature of $\mathrm{Tl}_{2} \mathrm{O}_{3}$ will be discussed further in relation to experimental data presented below. 

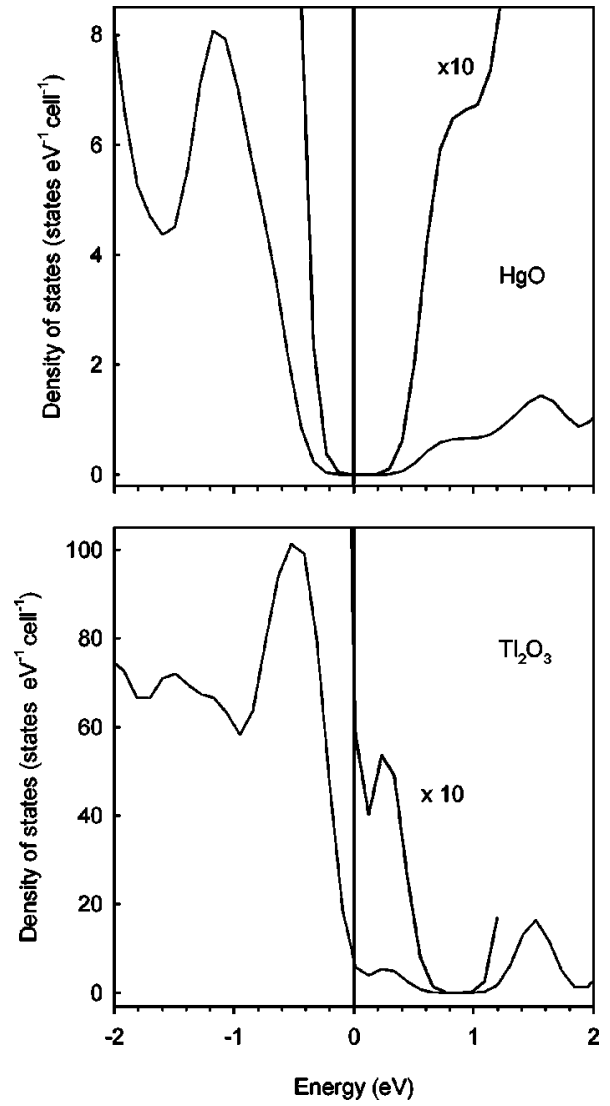

FIG. 7. Total densities of states for $\mathrm{HgO}$ and $\mathrm{Tl}_{2} \mathrm{O}_{3}$ in the vicinity of the Fermi energy. The metallic nature of $\mathrm{Tl}_{2} \mathrm{O}_{3}$ is evident from the nonzero value of the density of states at the Fermi energy.

\section{B. Valence region spectra of $\mathrm{HgO}$ : $\mathrm{Al} K \alpha$ x-ray photoemission and $O K$ shell $x$-ray emission}

The Al $K \alpha$ excited valence-band photoemission spectrum of $\mathrm{HgO}$ along with the corresponding $\mathrm{O} K$ shell x-ray emission spectrum are shown in Fig. 8. In each case, the spectral profiles may be fitted with five components, corresponding to bands I-V in the calculated density of states. The experimental data are compared in each case with theoretical spectral profiles. There is in general very good correspondence between the two, although the spread of energies found experimentally (about $10 \mathrm{eV}$ ) is slightly bigger than in the densityfunctional calculations (about $8 \mathrm{eV}$ ). "Compression" of the energy scale relative to that found experimentally appears to be a general feature of density-functional calculations. ${ }^{32}$

Consider first the XPS data. At high photon energies, it is generally recognized that the Gelius mode $\mathrm{l}^{40}$ provides a good description of intensities in valence-band photoemission spectra. The experimental spectral profile in Fig. 8(a) may therefore be compared with the cross-section weighted density of states $C(E)$ shown in Fig. 8(b), where

$$
C(E)=\sum_{i} \sigma_{i} N_{i}(E)
$$

Here the $\sigma_{i}$ are one-electron ionization cross sections, the $N_{i}(E)$ are partial densities of states, and the summation is taken across $\mathrm{Hg} 5 d, \mathrm{Hg} 6 s$, and $\mathrm{O} 2 p$ states. Because the cross section for ionization of $\mathrm{Hg} 5 d$ states at $h \nu$ $=1486.6 \mathrm{eV}$ is very much bigger than cross sections for $\mathrm{Hg}$ $6 s$ or O $2 p$ states,${ }^{41}$ the overall spectral profile is dominated by the $\mathrm{Hg} 5 d$ partial density of states. For this reason, the $5 d$ bands IV and V are much stronger than the $\mathrm{O} 2 p$ bands I-III. Moreover, band III is the strongest feature in the $\mathrm{O} 2 p$ region because of the strong contribution from $\mathrm{Hg} 5 d$ levels discussed above. Within the $5 d$ region, the experimental spectrum consists of what appears to be a simple spin-orbit doublet with an intensity ratio between bands IV and V of 2.73:2.27. It should also be noted that atomic Dirac-Fock calculations ${ }^{42}$ predict a $5 d_{5 / 2}: 5 d_{3 / 2}$ cross-section ratio of 2.89:2.11. This is less than the statistical value of $3: 2$ but nonetheless greater than the experimental value. Qualitatively, the observed deviation can be understood in terms of a stronger contribution from $\mathrm{O} 2 p$ states (which have a low cross section) to the less tightly bound $5 d_{5 / 2}$ states than to the more tightly bound $5 d_{3 / 2}$ states. This effect is reproduced in the calculations, although the $\sigma_{1 / 2}$ component of $5 d_{5 / 2}$ (which carries the greatest $\mathrm{O} 2 p$ contribution) is actually split out from the rest of the $5 d_{5 / 2}$ states and falls within the envelope of the $5 d_{3 / 2}$ states in band V.

Consider next the $\mathrm{O} K$ shell $\mathrm{x}$-ray emission spectra excited under nonresonant conditions shown in Fig. 8(c). Radiative decay of the $\mathrm{O} K$ shell hole states is governed by a strict dipole selection rule, which only allows transitions from $\mathrm{O} 2 p$ valence states. Thus the $\mathrm{x}$-ray emission data probe the $\mathrm{O} 2 p$ partial density of states. The calculated $\mathrm{O} 2 p$ partial density of states for $\mathrm{HgO}$ is therefore presented in Fig. 8(d) alongside the $\mathrm{O} K$ shell $\mathrm{x}$-ray emission data. Again, the experimental spectrum may be fitted to five peaks. In contrast to the $\mathrm{x}$-ray photoemission spectrum, the "O $2 p$ " peaks I-III have a higher intensity than the $\mathrm{Hg} 5 d$ peaks IV and V; since the intensity now depends on the $\mathrm{O} 2 p$ contribution to each peak, peak III no longer dominates the $\mathrm{O} 2 p$ region. Moreover, the fact that peaks IV and V can be observed at all is a reflection of mixing of $\mathrm{O} 2 p$ character in the $\mathrm{Hg} 5 d$ states. The intensity ratio between the $5 d_{5 / 2}$ peak IV and the $5 d_{3 / 2}$ peak $\mathrm{V}$ has a value of 3.2:1.8, which is now greater than the statistical ratio of $3: 2$. This provides further experimental confirmation of the fact that the less tightly bound $5 d_{5 / 2}$ level mixes more strongly with $\mathrm{O} 2 p$ states than the more tightly bound $5 d_{3 / 2}$ states, thus reversing the deviation from the statistical intensity ratio found in x-ray photoemission. Qualitatively, the excellent correspondence between the $\mathrm{Al} \mathrm{K \alpha}$ photoemission spectra and the cross-section weighted density of states and between the $\mathrm{O} K$ emission spectra and the $\mathrm{O} 2 p$ partial density of states provides striking confirmation of the importance of $\mathrm{Hg} 5 d$ mixing with $\mathrm{O} 2 p$ states in $\mathrm{HgO}$. Overall, the calculation appears to introduce a little too much $\mathrm{O} 2 p$ character into the $\mathrm{Hg} 5 d$ states and therefore the intensity within the $\mathrm{Hg} 5 d$ bands in the $\mathrm{O} 2 p$ partial density of states is stronger than the observed intensity in $\mathrm{O} K$ shell emission. At the same time (as already noted above), the calculations produce too big a splitting of the $\mathrm{Hg}$ states by the linear field. Ultimately, these minor quantitative discrepancies between theory and experiment may be traced to the fact that the separation of $\mathrm{Hg} 5 d$ from $\mathrm{O} 2 p$ is too small in the calculations and the interactions between the two levels are therefore too strong. 

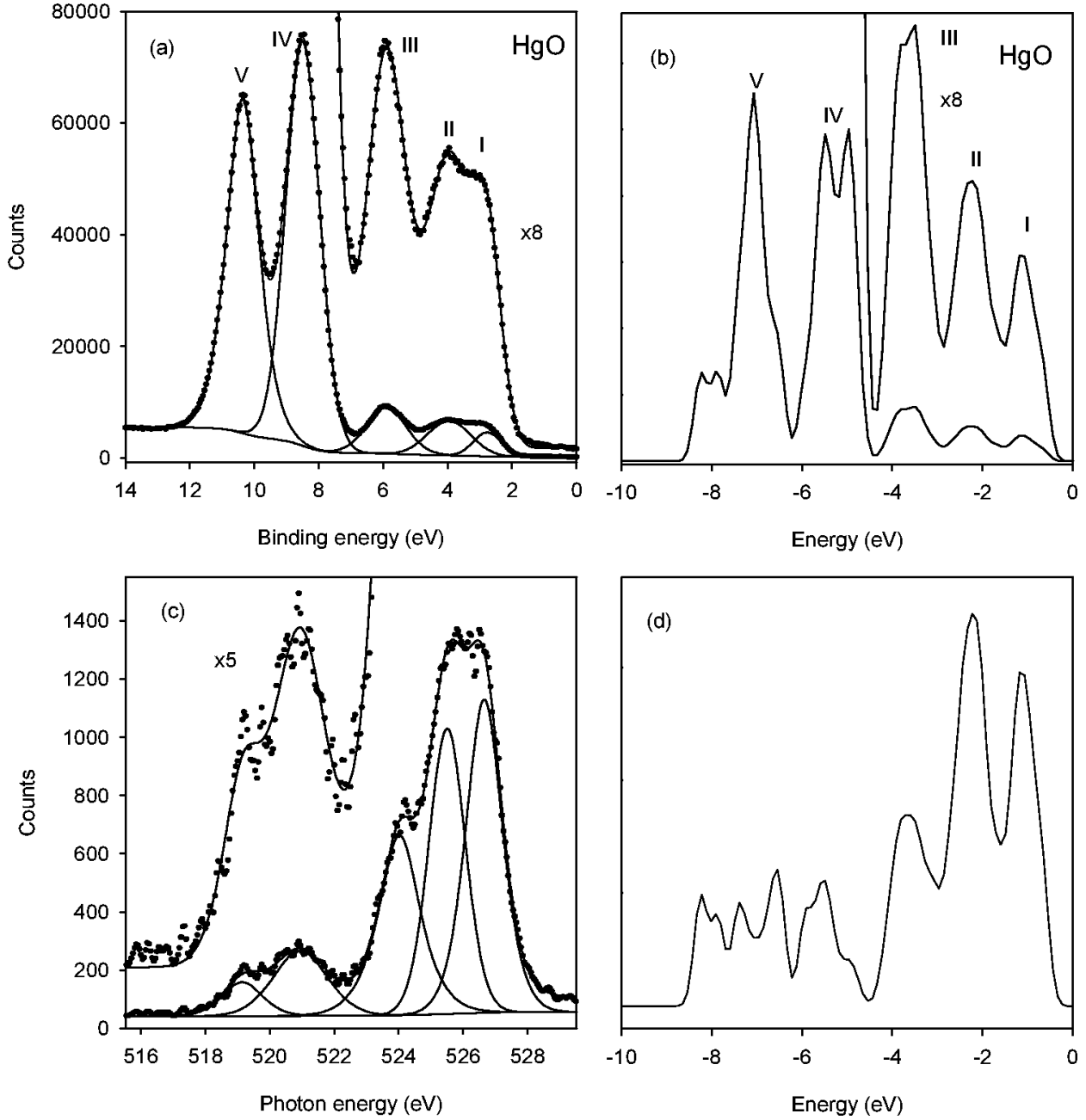

FIG. 8. (a) $\mathrm{Al} \quad K \alpha \quad(h \nu$ $=1486.6 \mathrm{eV}$ ) excited valenceband $\mathrm{x}$-ray photoemission spectrum of $\mathrm{HgO}$. (b) Cross-section weighted density of states for $\mathrm{HgO}$ derived from densityfunctional calculations. Ionization cross sections at $h \nu=1486.6 \mathrm{eV}$ are taken from the compilation of Yeh and Lindau. (c) Photon excited $(h \nu=570 \mathrm{eV})$ O $K$ shell $\mathrm{X}$-ray emission spectrum of $\mathrm{HgO}$. (d) $\mathrm{O} 2 p$ partial density of states for $\mathrm{HgO}$ derived from densityfunctional calculations.

\section{Valence region spectra of $\mathrm{Tl}_{2} \mathrm{O}_{3}$ : $\mathrm{Al} K \alpha$ x-ray photoemission and $O K$ shell $x$-ray emission}

The comparisons between $\mathrm{Al} K \alpha$ x-ray photoemission, $\mathrm{O} K$ shell $\mathrm{x}$-ray emission, and calculated densities of states for $\mathrm{Tl}_{2} \mathrm{O}_{3}$ are presented in Fig. 9 following the same format as the data for $\mathrm{HgO}$ in Fig. 8.

The x-ray photoemission data of Fig. 9(a) can again be discussed in terms of five peaks, where I-III correspond to $\mathrm{O} 2 p$ states and IV and V to Tl $5 d$ states. The overall spread of energies within the $\mathrm{O} 2 p$ region is bigger than in $\mathrm{HgO}$, with a distinct shift of the lowest-energy peak III to high binding energy as compared with $\mathrm{HgO}$. The intensity of the O $2 p$ peaks I-III relative to the Tl $5 d$ peaks IV and $\mathrm{V}$ is weaker by about a factor of 2 as compared with $\mathrm{HgO}$. This reflects the fact that there is less admixture of shallow core $5 d$ character into the $\mathrm{O} 2 p$ valence-band states. At the same time, the lowest band in the $\mathrm{O} 2 p$ region-band III-no longer dominates this region of the photoemission spectrum. This reflects the fact that shallow core $5 d$ character is no longer concentrated within this band.

A shift of the Tl $5 d$ shallow core levels to higher binding energy as compared with the $\mathrm{Hg} 5 d$ levels of $\mathrm{HgO}$ is observed. This is in accord both with simple periodic trends and with the calculations. Most surprisingly, however, the experimental metal $5 d$ peak shape appears more complex in
$\mathrm{Tl}_{2} \mathrm{O}_{3}$ than in $\mathrm{HgO}$ : an adequate fit to the shallow core region demands the introduction of two sets of overlapping $5 d_{5 / 2} / 5 d_{3 / 2}$ spin-orbit doublets, in contrast to the simple doublet which fits the data for $\mathrm{HgO}$. The additional structure within the $\mathrm{Tl} 5 d$ core levels is not a feature of the initial-state band structure but arises from final-state screening effects associated with a degenerate gas of mobile conduction electrons in metallic $\mathrm{Tl}_{2} \mathrm{O}_{3}$. The influence of the metallicity of $\mathrm{Tl}_{2} \mathrm{O}_{3}$ on core- and valence-level photoemission spectra will be discussed further in Sec. IV E. As with $\mathrm{HgO}$, the $5 d_{5 / 2}$ to $5 d_{3 / 2}$ intensity ratio has a value less than the statistical ratio of 3:2. In fact, the value found for $\mathrm{Tl}_{2} \mathrm{O}_{3}-2.70: 2.30$ - is slightly less than the value of 2.73:2.27 for $\mathrm{HgO}$. This is somewhat surprising given the more limited mixing between the metal $5 d$ and $\mathrm{O} 2 p$ levels in $\mathrm{Tl}_{2} \mathrm{O}_{3}$. Again the deviation cannot be explained in any simple way by purely atomic effects. The Dirac-Fock cross-section ratio has a value of 2.89:2.11, which is significantly bigger than that observed experimentally, and the photoelectron asymmetry parameters $\beta$ have very similar values for $5 d_{5 / 2}(\beta=1.34)$ and $5 d_{3 / 2}(\beta$ $=1.25$ ) levels. ${ }^{42}$

Nonresonant $\mathrm{O} K$ shell $\mathrm{x}$-ray emission data are shown in Fig. 9(c). Within the "O $2 p$ " region of bands I-III, it is particularly striking that the highest binding-energy band III is now much weaker than the two other $\mathrm{O} 2 p$ bands. This is because the states in band III have enhanced Tl $6 s$ atomic 

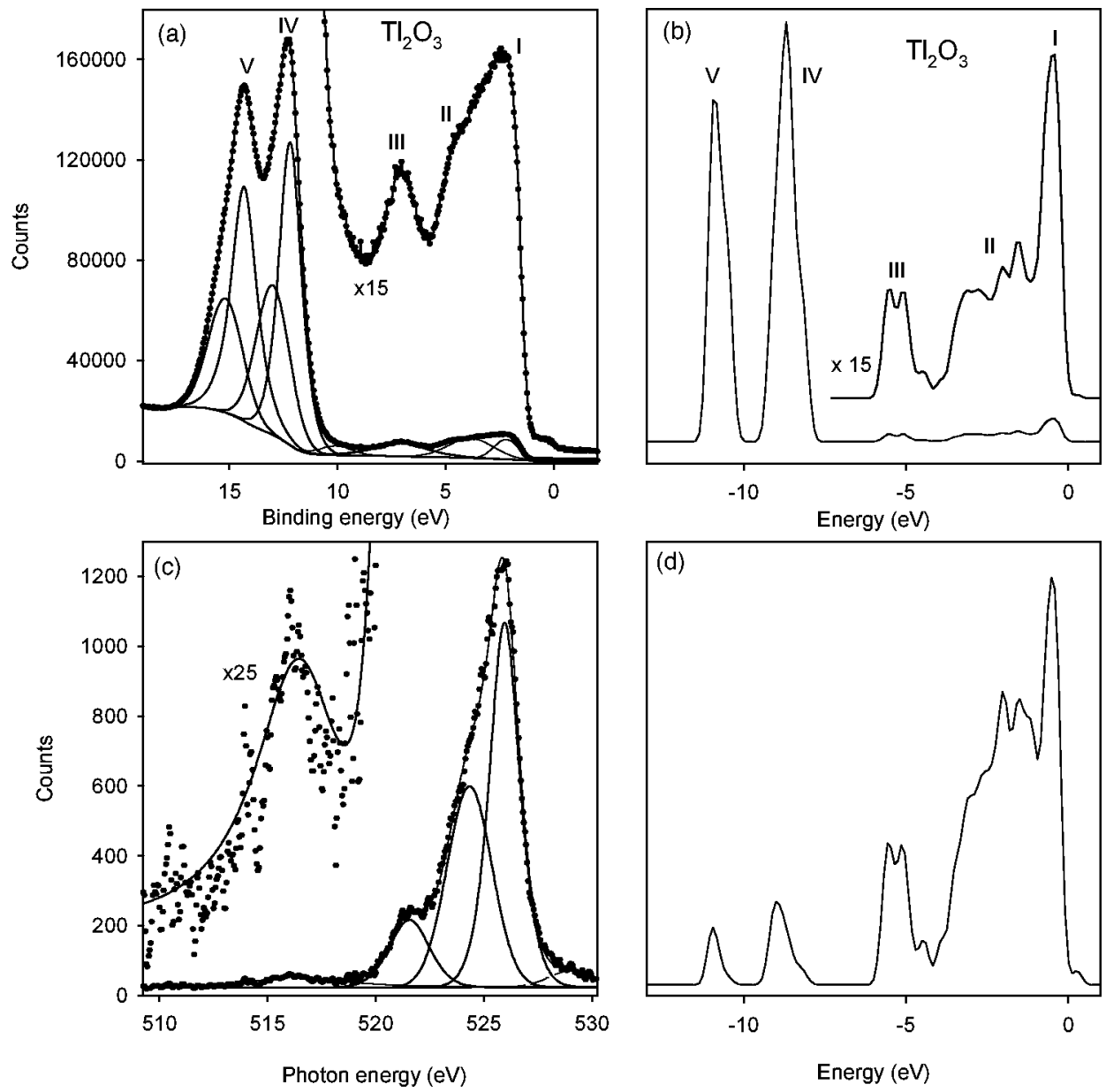

FIG. 9. (a) $\mathrm{Al} \quad K \alpha \quad(h \nu$ $=1486.6 \mathrm{eV})$ excited valenceband $\mathrm{x}$-ray photoemission spectrum of $\mathrm{Tl}_{2} \mathrm{O}_{3}$. (b) Cross-section weighted density of states for $\mathrm{Tl}_{2} \mathrm{O}_{3}$ derived from densityfunctional calculations. Ionization cross sections at $h \nu=1486.6 \mathrm{eV}$ are taken from the compilation of Yeh and Lindau. (c) Photon excited $(h \nu=570.5 \mathrm{eV}) \mathrm{O} K$ shell $\mathrm{x}$-ray emission spectrum of $\mathrm{Tl}_{2} \mathrm{O}_{3}$. (d) $\mathrm{O} 2 p$ partial density of states for $\mathrm{Tl}_{2} \mathrm{O}_{3}$ derived from densityfunctional calculations. character, with a corresponding reduction in the $\mathrm{O} 2 p$ contribution. Thus the $\mathrm{x}$-ray emission data provide confirmation of the buildup in metal $6 s$ character at the bottom of the valence band in going from $\mathrm{HgO}$ to $\mathrm{Tl}_{2} \mathrm{O}_{3}$. At the same time, the $\mathrm{Tl} 5 d$ structure in XES is very much weaker than the corresponding $\mathrm{Hg} 5 d$ structure for $\mathrm{HgO}$. Qualitatively, this is again in accord with the calculations, which predict much attenuated mixing between the shallow core $\mathrm{Tl} 5 d$ states and the $\mathrm{O} 2 p$ states. In fact, the experimental $\mathrm{Tl} 5 d$ structure excited under nonresonant conditions is so weak that it is not possible to fit two peaks with any statistical confidence. For this reason, the data are fitted to a single broad component which must, however, represent both the $5 d_{5 / 2}$ and the $5 d_{3 / 2}$ levels - the latter expected from the calculations to be weaker owing to the less pronounced mixing with $\mathrm{O} 2 p$.

\section{X-ray absorption and resonant $\mathrm{x}$-ray emission spectra of $\mathrm{HgO}$ and $\mathrm{Tl}_{2} \mathrm{O}_{3}$}

The $\mathrm{O} K$ shell $\mathrm{x}$-ray absorption spectrum of $\mathrm{HgO}$ is shown in Fig. 10 alongside $\mathrm{x}$-ray emission spectra excited at the series of photon energies superimposed on the absorption spectrum. At photon energies just above threshold, a distinct elastic scattering peak is observed above the main $\mathrm{O} 2 p$ valence-band edge in the emission spectra.

Pronounced variations in the relative intensities of the emission peaks with varying photon exciting energy are ap- parent. In particular, the $\mathrm{O} 2 p$ valence-band peak II grows in intensity with increasing photon energy and is strongest for photon energies of $534.25 \mathrm{eV}$ and $536.95 \mathrm{eV}$ : these energies are, respectively, $5.3 \mathrm{eV}$ and $8.0 \mathrm{eV}$ above threshold. This resonance effect appears to be related to the atomic nature of the states in the valence and conduction bands. The major $\mathrm{Hg}$ contribution to states in band II comes from $\mathrm{Hg} 6 p$ rather than $\mathrm{Hg} 6 s$. The dominant $\mathrm{Hg}$ contribution to states at the bottom of the conduction band is from $6 s$ states: $\mathrm{Hg} 6 p$ character is found in the empty states between $4 \mathrm{eV}$ and $10 \mathrm{eV}$ above threshold, as shown in Fig. 10. Thus the makeup of the states enhanced in emission seems to mirror that of the states to which the electron is excited in absorption, with both having strongly mixed $\mathrm{Hg} 6 p / \mathrm{O} 2 p$ atomic character.

The corresponding $\mathrm{O} K$ shell $\mathrm{x}$-ray absorption spectrum of $\mathrm{Tl}_{2} \mathrm{O}_{3}$ is shown in Fig. 11 alongside $\mathrm{x}$-ray emission spectra excited at the series of photon energies superimposed on the absorption spectrum. In contrast to $\mathrm{HgO}$, an elastic scattering peak is not visible. Instead, under photon excitation just above the $\mathrm{O} 1 s$ core threshold, distinct structure is visible above the main $\mathrm{O} 2 p$ structure. This arises from states associated with the metallic conductivity of $\mathrm{Tl}_{2} \mathrm{O}_{3}$ and is discussed in greater detail in Sec. IV F. The intensity of this structure decreases with increasing photon energy.

The intensity of $\mathrm{Tl} 5 d$ level emission is seen to exhibit pronounced variation with photon energy. Just above the onset of $\mathrm{O} K$ shell absorption at $h \nu=528.0 \mathrm{eV}, 5 d$ emission is not discernible above the background. The $5 d$ intensity in- 

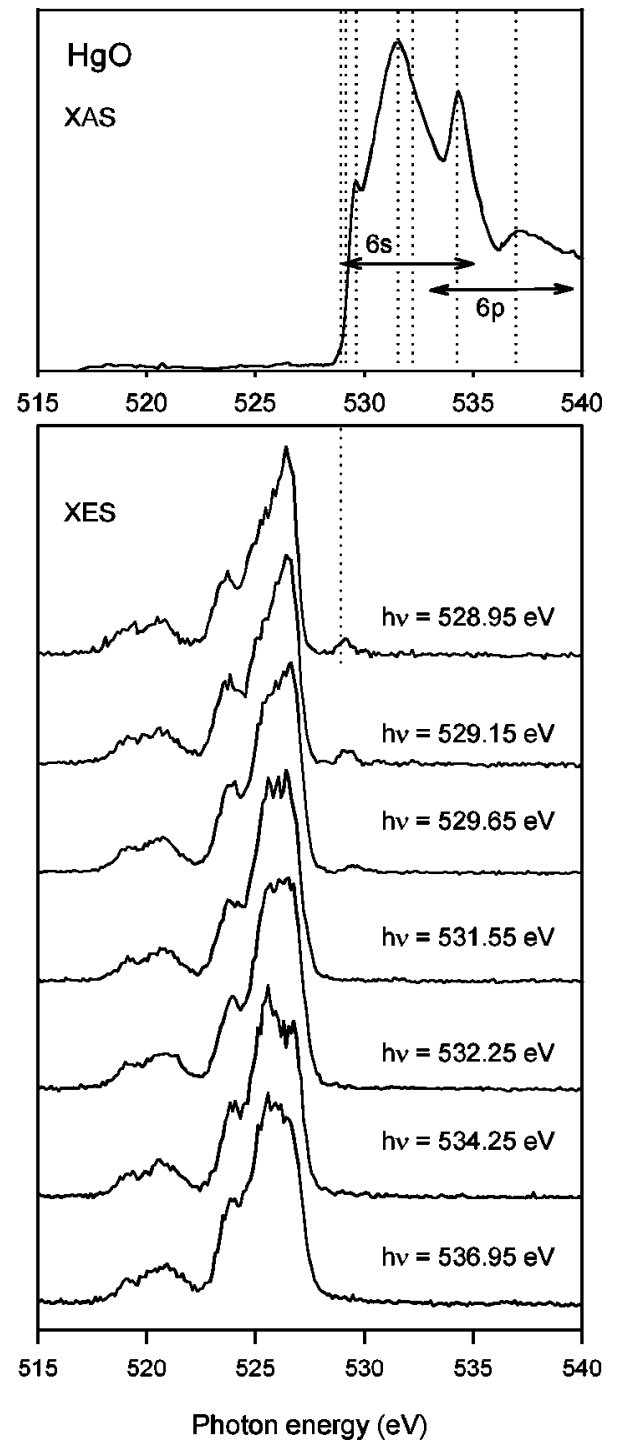

FIG. 10. Upper panel: $\mathrm{x}$-ray absorption spectrum of $\mathrm{HgO}$ in the region of the $\mathrm{O} 1 s$ core threshold. The vertical dotted lines delineate photon energies at which emission spectra have been excited in the data presented below. The regions with significant $\mathrm{Hg} 6 s$ and $\mathrm{Hg} 6 p$ contribution to the empty states are indicated by double-headed arrows. Lower panel: $\mathrm{x}$-ray emission spectra of $\mathrm{HgO}$ excited at the photon energies indicated. The threshold photon energy is indicated by a vertical dashed line.

creases with increasing photon energy and well-defined structure is visible at $h \nu=531.8 \mathrm{eV}$. The structure is even stronger at $h \nu=536.3 \mathrm{eV}$. At this photon energy, both components of the $5 d$ spin-orbit doublet are clearly visible: a pronounced deviation of the intensity ratio of $5 d_{5 / 2}$ and $5 d_{3 / 2}$ peaks below the statistical value of 3:2 is apparent. In fact, curve fitting the Tl $5 d$ peaks excited at $h \nu=536.3 \mathrm{eV}$ gives a $5 d_{5 / 2}$ to $5 d_{3 / 2}$ intensity ratio close to $4.0: 1.0$. This confirms that $\mathrm{Tl} 5 d_{5 / 2}$ mixes more strongly with $\mathrm{O} 2 p$ states than does Tl $5 d_{3 / 2}$, as suggested by the calculations.

\section{E. $\mathrm{Hg} N_{6,7}$ emission spectra of $\mathrm{HgO}$}

Emission spectra following generation of a core hole in the $\mathrm{Hg} 4 f$ core levels $\left(\mathrm{Hg} N_{6,7}\right)$ provide an additional ap-
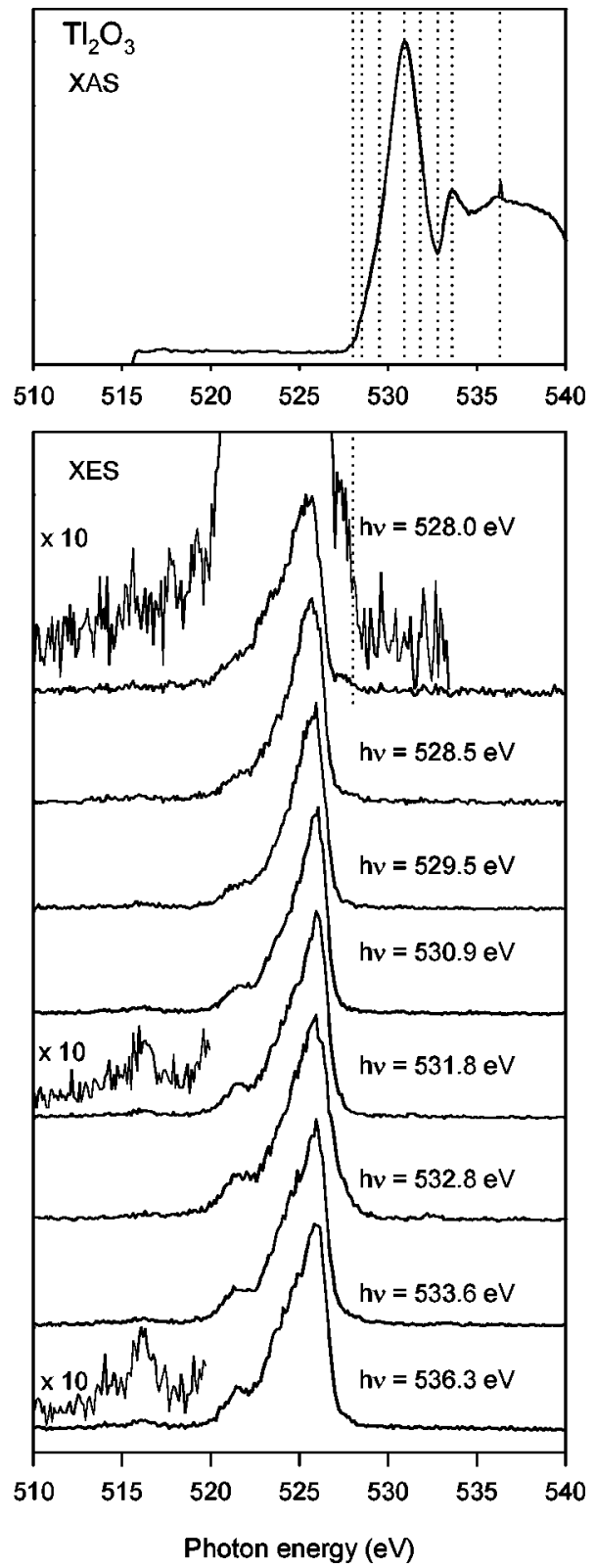

FIG. 11. Upper panel: $x$-ray absorption spectrum of $\mathrm{Tl}_{2} \mathrm{O}_{3}$ in the region of the $\mathrm{O} 1 s$ core threshold. The vertical dashed lines delineate photon energies at which emission spectra have been excited in the data presented below. Lower panel: $\mathrm{x}$-ray emission spectra of $\mathrm{Tl}_{2} \mathrm{O}_{3}$ excited at the photon energies indicated. The threshold photon energy is indicated by a vertical dashed line.

proach to probing mixing between $\mathrm{Hg} 5 d$ and $\mathrm{O} 2 p$ states. Before discussing the $N_{6,7}$ absorption and emission spectra, it is necessary to consider the $\mathrm{Hg} 4 f$ structure in XPS shown in Fig. 12. For completeness, this figure also includes the $\mathrm{Tl} 4 f$ core-level spectrum of $\mathrm{Tl}_{2} \mathrm{O}_{3}$. As expected, the $\mathrm{Hg}$ states give a simple spin-orbit doublet. The $\mathrm{Hg} 4 f_{7 / 2}$ and $\mathrm{Hg} 4 f_{5 / 2}$ binding energies are $100.57 \mathrm{eV}$ and $104.61 \mathrm{eV}$, respectively. The corresponding $N_{6,7}$ absorption spectrum shown in Fig. 13, therefore, contains two well-separated peaks associated with the absorption thresholds for the two components of the $4 f$ level. Emission spectra were measured under excitation with the range of photon energies depicted in the upper panel of 

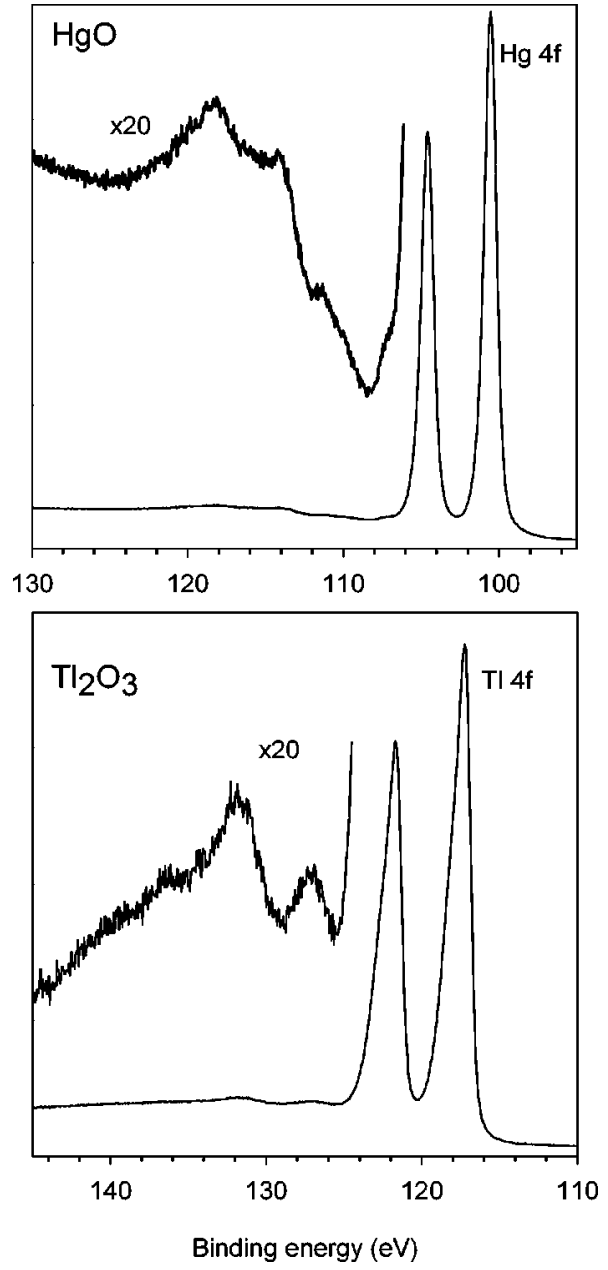
$\mathrm{Tl}_{2} \mathrm{O}_{3}$

FIG. 12. $4 f$ core level x-ray photoelectron spectra of $\mathrm{HgO}$ and

Fig. 13. At high incident photon energies, the overall emission spectrum is a superposition of structure arising from radiative decay into both the $4 f_{7 / 2}\left(N_{7}\right)$ and $4 f_{5 / 2}\left(N_{6}\right)$ levels. The dominant contribution to the intensity obviously comes from $\mathrm{Hg} 5 d$ to $\mathrm{Hg} 4 f$ transitions. However, the separation of $3.29 \mathrm{eV}$ between the two strong peak maxima in the emission spectrum excited at $112.00 \mathrm{eV}$ is significantly less than the separation of $4.04 \mathrm{eV}$ between $4 f_{7 / 2}$ and $4 f_{5 / 2}$ observed in core-level photoemission. This is because of the operation of a $\Delta J=0, \pm 1$ selection rule, which allows transitions only from $5 d_{5 / 2}$ into $4 f_{7 / 2}$ but from both $5 d_{5 / 2}$ and $5 d_{3 / 2}$ into $4 f_{5 / 2}$. Although the spin-orbit splitting of the $5 d$ shell is not resolved in the $\mathrm{Hg} N_{6}$ spectrum, the contributions to the peak from $5 d_{3 / 2}$ transitions must act to shift spectral weight to lower photon energy in the $N_{6}$ emission peak, thus decreasing the separation between the $N_{7}$ and $N_{6}$ peak maxima compared with the value observed in photoemission.

By selecting photon energies below the $N_{6}$ threshold, emission spectra free of structure due to decay into the $N_{6}$ hole may be obtained. Thus the spectra excited at $100.55 \mathrm{eV}$ and $103.20 \mathrm{eV}$ are much simpler than those excited at higher photon energy and contain a single strong peak due to transitions from the $\mathrm{Hg} 5 d$ level. The observation of a peak labeled $b$ in the $100.55 \mathrm{eV}$ spectrum in addition to the domi-
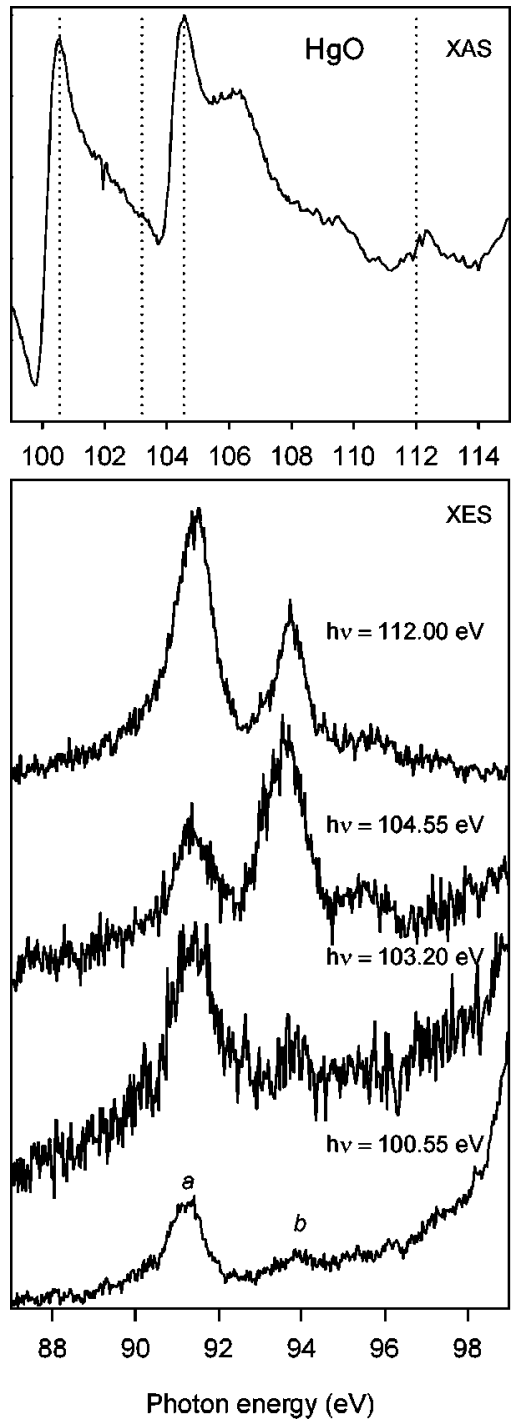

FIG. 13. $\mathrm{Hg} N_{6,7}(4 f)$ x-ray emission structure for $\mathrm{HgO}$.

nant peak $a$ is due to radiative transitions into the $N_{7}$ hole from the most tightly bound "O $2 p$ " valence-band state, labeled as peak III in the discussion of XPS data above. The separation between $a$ and $b$ observed in the emission spectrum has a value of $2.2 \mathrm{eV}$, which is close to the separation of $2.5 \mathrm{eV}$ observed in photoemission. Observation of peak $b$ is only possible by virtue of $\mathrm{Hg} 5 d$ admixture into the relevant "O $2 p$ " state. Thus the $N_{7}$ emission data provide additional confirmation of the pronounced $\mathrm{Hg} 5 d$ character in the most tightly bound "O $2 p$ " state.

A final feature of interest in relation to the $N_{6,7}$ emission spectra is the enhancement of the intensity of $N_{6}$ emission just above the $N_{6}$ absorption threshold (104.55 eV) as compared with the higher photon energy of $112.00 \mathrm{eV}$.

\section{F. The band gap of $\mathrm{HgO}$ and the metallic nature of $\mathrm{Tl}_{2} \mathrm{O}_{3}$}

Examination of structure in x-ray photoemission, $\mathrm{x}$-ray absorption, and $\mathrm{x}$-ray emission spectra in the vicinity of the Fermi energy provides further insight into the differences in electronic structure between $\mathrm{HgO}$ and $\mathrm{Tl}_{2} \mathrm{O}_{3}$. The photoemis- 

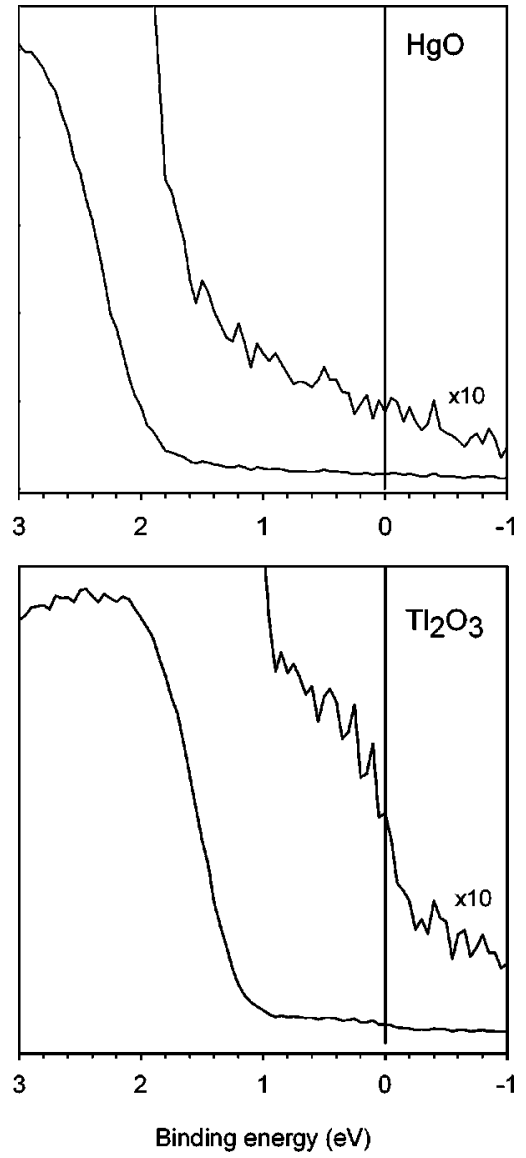

FIG. 14. Expansion of the low-energy region in Al $K \alpha$ ( $h \nu$ $=1486.6 \mathrm{eV}$ ) excited valence band x-ray photoemission of (a) $\mathrm{HgO}$ and (b) $\mathrm{Tl}_{2} \mathrm{O}_{3}$. Note the metallic Fermi edge onset for the latter.

sion onsets over an energy range of $4 \mathrm{eV}$ around the Fermi energy are shown in Fig. 14.

$\mathrm{HgO}$ displays a spectrum typical of a nonmetallic material, with only weakly increasing intensity below the Fermi energy until the onset of the main $\mathrm{O} 2 p$ valence-band edge. Linear extrapolation of this edge suggests a threshold of about $1.9 \pm 0.1 \mathrm{eV}$. This is compatible with the literature value of the bulk band gap for $\mathrm{HgO}$ if it is assumed that the Fermi energy is pinned by states at the bottom of the bulk conduction band. The shift between onsets for x-ray absorption and $\mathrm{x}$-ray emission under threshold excitation shown in Fig. 15 suggests a slightly lower value of perhaps $1.7 \mathrm{eV}$ for the bulk band gap. However, the signal to noise in these spectra is not good and so it is not possible to determine onset energies precisely.

By contrast, the photoemission spectrum of $\mathrm{Tl}_{2} \mathrm{O}_{3}$ displays a distinct metallic Fermi edge. Of course this accords with the bulk transport properties. However, the Fermi energy does not coincide with the edge of the main valence band, but lies in a weak band which extends to higher energy. The main valence-band onset is about $1.1 \mathrm{eV}$ below the Fermi energy. Thus although photoemission results confirm the metallic behavior emerging from band-structure calculations, the position of the Fermi energy is different in detail from that in the calculations. The experimental results thus suggest that extra electrons must be introduced into the sys-
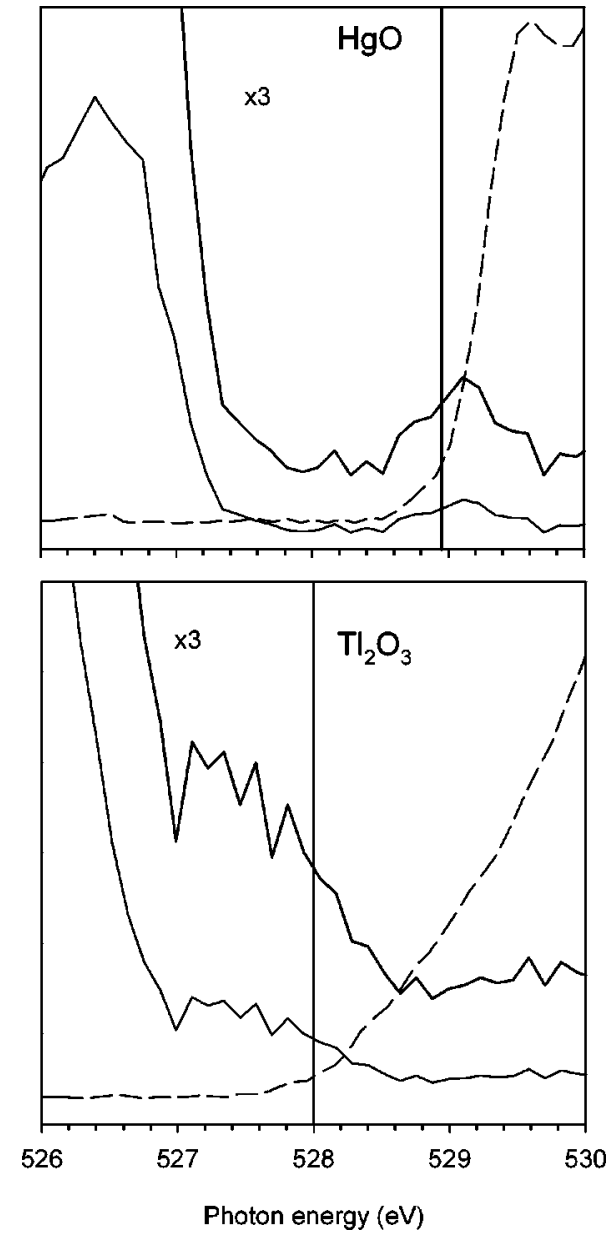

FIG. 15. Expansions of x-ray absorption spectra (dashed lines) and threshold x-ray emission spectra of $\mathrm{HgO}$ (upper panel) and $\mathrm{Tl}_{2} \mathrm{O}_{3}$ (lower panel). The vertical lines delineate the photon energy used to excite the threshold emission spectra.

tem by some sort of $n$-type doping, thus partially filling the weak band of states above the Fermi energy revealed in the band-structure calculations. The doping could arise from oxygen deficiency in $\mathrm{Tl}_{2} \mathrm{O}_{3-x}$, each oxygen vacancy being associated with two electrons. The $\mathrm{O} K$ shell $\mathrm{x}$-ray emission spectrum of $\mathrm{Tl}_{2} \mathrm{O}_{3}$ excited at the $\mathrm{O} 1 s$ core threshold provides a complementary picture of the density of states in the vicinity of the Fermi energy (Fig. 15). Again, a weak band of states is observed immediately below the Fermi energy with the main valence-band onset about $1 \mathrm{eV}$ below the Fermi level. Of course, the appearance of the states above the valence band in $\mathrm{O} K$ shell $\mathrm{x}$-ray emission implies that the states must have substantial $\mathrm{O} 2 p$ character. This is at variance with the simplest ionic picture but is in accord with the strong O $2 p$-Tl $6 s$ mixing revealed by the calculations. Taken together, the x-ray absorption and emission data help to explain why $\mathrm{Tl}_{2} \mathrm{O}_{3}$ is quoted to have an apparent band gap of $1.6 \mathrm{eV}$. As we have seen, the main valence-band edge is about $1.1 \mathrm{eV}$ below the Fermi energy. The density of empty states increases only very slowly above the Fermi energy. Thus the onset of strong optical absorption is not observed until the photon energy reaches $1.6 \mathrm{eV}$. 


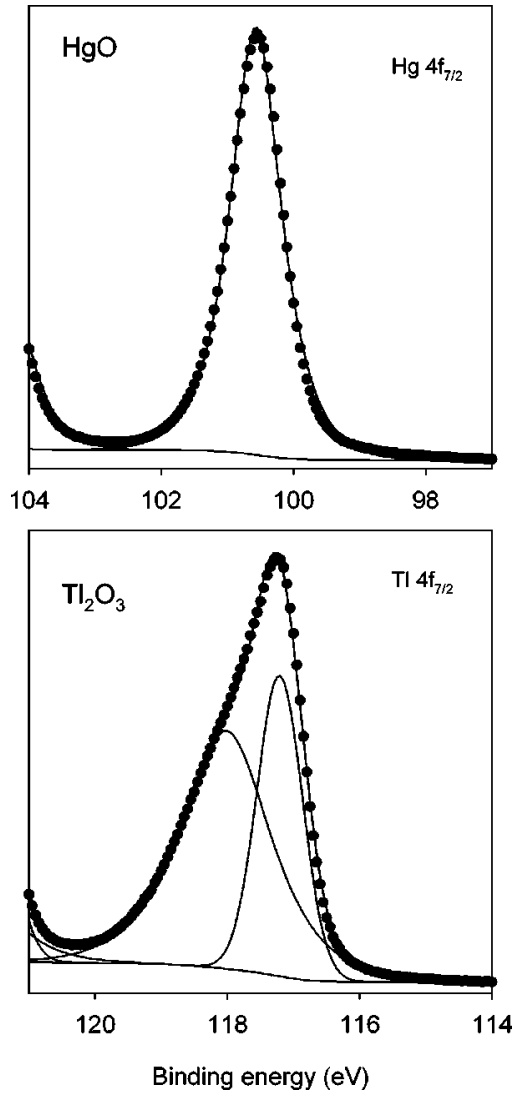

FIG. 16. Al $K \alpha(h \nu=1486.6 \mathrm{eV})$ excited $4 f_{7 / 2}$ core-level structure for $\mathrm{HgO}$ and $\mathrm{Tl}_{2} \mathrm{O}_{3}$. The strong satellite structure in the latter is associated with plasmon excitation.

\section{G. Satellite structure in core-level photoemission from $\mathrm{Tl}_{2} \mathrm{O}_{3}$}

As discussed above, the $\mathrm{Hg} 4 f$ photoemission spectrum of $\mathrm{HgO}$ comprises a simple spin-orbit doublet. Each of the spinorbit components may be fitted by a simple pseudo-Voigt function with a FWHM just over $0.9 \mathrm{eV}$ : the fit for the $4 f_{7 / 2}$ component is shown in Fig. 16. By contrast, the $\mathrm{Tl} 4 f$ core line shape for $\mathrm{Tl}_{2} \mathrm{O}_{3}$ is more complex, with distinct shoulders on the high-binding-energy side of both peaks. The overall Tl $4 f$ spectral profile may be fitted with two pairs of spinorbit doublets: the fit to the $4 f_{7 / 2}$ component is also shown in Fig. 16, while the parameters derived from the peak fit are given in Table III. The high-binding-energy components responsible for the shoulders are much broader than the "main" low-binding-energy peaks and carry greater spectral weight. It is also striking that the high-binding-energy satellites have a dominantly Lorentzian line shape. The separation $\Delta_{\text {sat }}$ of the high-binding-energy satellites from the main peak is $0.81 \mathrm{eV}$ for both the $4 f_{7 / 2}$ and $4 f_{5 / 2}$ components. Similar satellite energies are derived from curve fitting the $5 d$ spectrum (Table III).

The core photoemission spectra of $\mathrm{Tl}_{2} \mathrm{O}_{3}$ are very similar to those of other metallic post-transition metal oxides including Sb-doped $\mathrm{SnO}_{2}$ (Refs. 43 and 44) and Sn-doped $\mathrm{In}_{2} \mathrm{O}_{3} \cdot{ }^{45}$ The observation of strong satellites for $\mathrm{Tl}_{2} \mathrm{O}_{3}$ but not for $\mathrm{HgO}$ is obviously a direct consequence of the metallic nature of the former. The simplest model is that the broad highenergy peaks correspond to unusually strong conduction
TABLE III. Binding energies, halfwidths, and relative intensities for $4 f$ and $5 d$ core levels in $\mathrm{HgO}$ and $\mathrm{Tl}_{2} \mathrm{O}_{3} . S$ and $U$ in the first column indicate screened and unscreened final states.

\begin{tabular}{lcccccc}
\hline \hline & $\begin{array}{c}\mathrm{BE} \\
(\mathrm{eV})\end{array}$ & $\begin{array}{c}\Delta_{\text {SO }} \\
(\mathrm{eV})\end{array}$ & $\begin{array}{c}\Delta_{\text {sat }} \\
(\mathrm{eV})\end{array}$ & $\begin{array}{c}\text { FWHM } \\
(\mathrm{eV})\end{array}$ & $\begin{array}{c}\text { Relative } \\
\text { intensity }\end{array}$ & $\begin{array}{c}\text { Relative } \\
\text { intensity }\end{array}$ \\
\hline $\mathrm{Hg} \mathrm{4f_{7/2 }}$ & 100.57 & & & 0.91 & 4.00 & \\
$\mathrm{Hg} \mathrm{4f_{5/2 }}$ & 104.61 & 4.04 & & 0.92 & 3.00 & \\
$\mathrm{Hg} \mathrm{5} d_{5 / 2}$ & 8.50 & & & 1.21 & 2.73 & \\
$\mathrm{Hg} \mathrm{5} d_{3 / 2}$ & 10.36 & 1.86 & & 1.15 & 2.27 & \\
$\mathrm{Tl} 4 f_{7 / 2} S$ & 117.21 & & & 0.81 & 4.00 & 1.48 \\
$\mathrm{Tl} 4 f_{7 / 2} U$ & 118.02 & & 0.81 & 1.58 & & 2.52 \\
$\mathrm{Tl} 4 f_{5 / 2} S$ & 121.64 & 4.43 & & 0.82 & 3.00 & 1.13 \\
$\mathrm{Tl} 4 f_{5 / 2} U$ & 122.45 & 4.43 & 0.81 & 1.57 & & 1.87 \\
$\mathrm{Tl} 5 d_{5 / 2} S$ & 12.20 & & & 1.22 & 2.70 & 1.59 \\
$\mathrm{Tl} 5 d_{5 / 2} U$ & 13.02 & & 0.82 & 1.82 & & 1.11 \\
$\mathrm{Tl} 5 d_{3 / 2} S$ & 14.33 & 2.13 & & 1.39 & 2.30 & 1.45 \\
$\mathrm{Tl} 5 d_{3 / 2} U$ & 15.17 & 2.15 & 0.84 & 1.87 & & 0.85 \\
\hline \hline
\end{tabular}

Intensities are normalized such that the total intensity is 7 for $4 f$ levels and 5 for $5 d$ levels.

${ }^{b}$ The total intensity for the spin-orbit components in $\mathrm{Tl}_{2} \mathrm{O}_{3}$ is broken down into contributions from screened and unscreened final states.

electron plasmon satellites. The separation of the "satellite" from the main peak- $0.81 \mathrm{eV}$ - is in fact very similar to the plasmon energy of $0.76 \mathrm{eV}$ derived from analysis of reflection spectra of $\mathrm{Tl}_{2} \mathrm{O}_{3}$ thin films. ${ }^{22}$

It was recognized early in the application of photoemission techniques to simple metallic solids that plasmon satellites make a significant contribution to core-level structure in $\mathrm{x}$-ray photoelectron spectra. Much of the early work in this area was concerned with unraveling the relative contributions of intrinsic and extrinsic structure and with rationalizing the pattern of multiple plasmon excitations. ${ }^{46}$ The weakcoupling models developed 30 years ago ${ }^{47}$ suggested that the intrinsic plasmon satellite intensity $I$ should increase as the conduction electron density $n$ decreases according to the expression $I \propto N^{-1 / 3}$. Thus within the framework of this model, the high intensity of the satellites observed in the present work reflects the low density of conduction electrons. The plasmon energy is determined by the carrier concentration $N$ through the relationship

$$
\omega_{p}^{2}=\frac{N e^{2}}{m^{*} \varepsilon_{0} \varepsilon(\infty)},
$$

where $\omega_{p}$ is the plasmon frequency, $m^{*}$ is the effective mass, and $\varepsilon(\infty)$ is the high-frequency dielectric constant. The analysis of reflectivity data by Geserich $^{22}$ gives a value of $0.32 m_{0}$ for the effective mass and $\varepsilon(\infty) \approx 5$. This suggests that the carrier concentration in our sample after gentle UHV annealing must be about $7.6 \times 10^{20} \mathrm{~cm}^{-3}$. This is of the same order as found in reactively sputtered thin films or in single crystals annealed at $550{ }^{\circ} \mathrm{C}$ in $2 \times 10^{4} \mathrm{~Pa}$ oxygen pressure. The carrier concentration in turn equates to an oxygen deficiency parameter $x=9.6 \times 10^{-3}$ defined by the formula 
$\mathrm{Tl}_{2} \mathrm{O}_{3-x}$, assuming that oxygen vacancies are doubly ionized. A small degree of oxygen deficiency of this sort seems quite reasonable.

An obvious problem with the plasmon model is that the abnormally high satellite intensity calls into question the applicability of a weak-coupling model in the first place. Moreover, in "simple" metals the overall line shape involves multiple plasmon loss satellites, whereas in the present work only a single satellite is observed. Similar behavior has been observed in core photoemission of a wide-range "narrowband" metallic transition-metal oxides including the sodium tungsten bronzes $\mathrm{Na}_{x} \mathrm{WO}_{3},{ }^{48-51}$ the superconducting spinel $\mathrm{LiTi}_{2} \mathrm{O}_{4},{ }^{52,53}$ dioxides such as $\mathrm{MoO}_{2}$ (Ref. 53) and $\mathrm{RuO}_{2},{ }^{54}$ and the ternary metallic pyrochlore ruthenates $\mathrm{Pb}_{2} \mathrm{Ru}_{2} \mathrm{O}_{7}$ and $\mathrm{Bi}_{2} \mathrm{Ru}_{2} \mathrm{O}_{7} .{ }^{55}$ Satellite energies for these materials are all around $1-2 \mathrm{eV}$. Wertheim ${ }^{48-50,50}$ suggested an alternative limiting model for the satellites in this family of narrowband metals. This involves a screening mechanism in which the Coulomb potential of the core hole at an ionized atom creates a localized trap state. In this situation, two different final states are then accessible depending on whether the localized state remains empty (giving an unscreened states) or is filled by transfer of an electron from the conduction band (to give a screened final state). In the model developed by Kotani and Toyazawa, ${ }^{56}$ the screened final state gives rise to an asymmetric line to low binding energy of the lifetime broadened peak associated with the unscreened final state. In the alternative language of the plasmon model, the high-bindingenergy unscreened peak corresponds to an extrinsic plasmon satellite whose Lorentzian linewidth will be determined by the conduction electron scattering rate. Whichever model is preferred, it is interesting to note that the appearance of lowenergy screening satellites at $0.81 \mathrm{eV}$ for $\mathrm{Tl}_{2} \mathrm{O}_{3}$ is accompanied by a striking decrease in the intensity of the broad satellite structure at higher energy as compared with $\mathrm{HgO}$ (Fig. 12). This suggests that the spectral weight associated with final-state screening is redistributed into the low-energy satellites.

\section{CONCLUDING REMARKS}

The combination of XPS, XAS, and XES with densityfunctional calculations allows us to develop a consistent picture of the electronic structure of $\mathrm{HgO}$ and $\mathrm{Tl}_{2} \mathrm{O}_{3}$. Both are highly covalent materials with strong mixing between metal $6 s$ and $6 p$ states and $\mathrm{O} 2 p$ states. In addition, for $\mathrm{HgO}$ there is very strong mixing between shallow core $\mathrm{Hg} 5 d$ states and O $2 p$ states, the mixing of $\mathrm{Hg} 5 d_{5 / 2}$ being more pronounced than that of $\mathrm{Hg} 5 d_{3 / 2}$. The consequence of mixing of both $\mathrm{Hg}$ $6 s$ and $5 d$ states with $\mathrm{O} 2 p$ states is that the bottom of the $\mathrm{O}$ $2 p$ valence band acquires pronounced $\mathrm{Hg} 6 s$ and $\mathrm{Hg} 5 d$ character simultaneously. There is, however, little direct mixing between filled $\mathrm{Hg} 5 d$ states and empty $\mathrm{Hg} 6 s$ conductionband states in the way described in the conventional textbook explanation of the liner stereochemistry of $\mathrm{Hg}$ II. The situa- tion is in some ways similar to that for PbO. Here the asymmetric coordination environment found for $\mathrm{Pb}$ is conventionally attributed to direct intra-atomic mixing between the occupied $\mathrm{Pb} 6 s$ level and an empty $\mathrm{Pb} 6 p$ level. This mixing becomes possible only if the $\mathrm{Pb}$ ions occupy a noncentrosymmetric site and is expected to give rise to an asymmetric electron density around $\mathrm{Pb}$. However, we have recently shown ${ }^{57,58}$ that the $\mathrm{Pb} 6 s$ states mix strongly with $\mathrm{O} 2 p$ states to give a lower in-phase combination of dominant $\mathrm{Pb} 6 s$ character and an upper out-of-phase combination of dominant $\mathrm{O} 2 p$ character. The asymmetric electron density derives mainly from mixing of the latter with the empty $\mathrm{Pb} 6 p$ levels, rather than from direct on-site mixing between $\mathrm{Pb} 6 s$ and $\mathrm{Pb} 6 p$ states.

Mixing between shallow core $5 d$ levels and $\mathrm{O} 2 p$ valence levels is less pronounced in $\mathrm{Tl}_{2} \mathrm{O}_{3}$ than in $\mathrm{HgO}$, but is not negligible. Again differential mixing between $5 d_{5 / 2}$ and $5 d_{3 / 2}$ levels may be inferred both from $\mathrm{O} K$ shell emission intensities and the band-structure calculations.

Demonstration of metal $5 d / \mathrm{O} 2 p$ mixing in the present work implies that states in the $\mathrm{O} 2 p$ valence band in cuprate superconductors containing $\mathrm{Hg}$ or $\mathrm{Tl}$ should have some metal $5 d$ character. Thus $p$-type doping must involve introduction of a hole character at the $\mathrm{Hg}$ or $\mathrm{Tl}$ sites. This hitherto overlooked effect provides a mechanism for introduction of a weak but possibly important electronic coupling between the doped $\mathrm{CuO}_{2}$ sheets. At the same time, the differential mixing of the $5 d_{5 / 2}$ and $5 d_{3 / 2}$ spin-orbit levels implies spin polarization in the $\mathrm{O} 2 p$ states. The possible impact of these effects on superconductivity in cuprate phases deserves further investigation.

Finally, the present study helps clarify the origin of metallic conductivity in $\mathrm{Tl}_{2} \mathrm{O}_{3}$. Band-structure calculations suggest that a single band disperses across the Fermi energy and that stoichiometric $\mathrm{Tl}_{2} \mathrm{O}_{3}$ should therefore be metallic with a Fermi level near the top of the $\mathrm{O} 2 p$ valence band. Experimentally, the Fermi level is in fact found in a weak band which is truncated by a Fermi-Dirac cutoff about $1.1 \mathrm{eV}$ above the main valence-band edge. This in turn implies that oxygen vacancies introduce extra carriers into the system. Analysis of plasmon structure in core photoemission spectra gives a value of about $7.6 \times 10^{20} \mathrm{~cm}^{-3}$ for the effective carrier concentration, a value consistent with previous work on $\mathrm{Tl}_{2} \mathrm{O}_{3}$ thin films and crystals.

\section{ACKNOWLEDGMENTS}

We are grateful to the UK EPSRC for support of the NCESS facility. The Boston University program is supported in part by the U.S. Department of Energy under Contract No. DE-FG02-98ER45680. The ALS is supported by the U.S. Department of Energy, Materials Sciences Division, under Contract No. DE-AC03-76SF00098 at Lawrence Berkeley National Laboratory. The Trinity College Dublin program is supported by the HEA. 
*Corresponding author. Email address: russ.egdell@chem.ox.ac.uk

'Permanent address: Department of Chemistry, University of Modena and Reggio Emilia, 41100 Modena, Italy.

${ }^{1}$ Z. Z. Sheng and A. M. Herman, Nature (London) 332, 138 (1988).

${ }^{2}$ T. R. Askew and R. B. Flippen, Science 242, 249 (1988).

${ }^{3}$ S. N. Putilin, E. V. Antipov, O. Chmaissem, and M. Marezio, Nature (London) 362, 226 (1993).

${ }^{4}$ A. Schilling, M. Cantoni, J. D. Guo, and H. R. Ott, Nature (London) 363, 56 (1993).

${ }^{5}$ L. Gao, Y. Y. Xue, F. Chen, Q. Xiong, R. L. Meng, D. Ramirez, C. W. Chu, J. H. Eggert, and H. K. Mao, Phys. Rev. B 50, R4260 (1994).

${ }^{6}$ A. R. Armstrong, W. I. F. David, I. Gameson, P. P. Edwards, J. J. Capponi, P. Bordet, and M. Marezio, Phys. Rev. B 52, 15551 (1995).

${ }^{7}$ A. R. Armstrong and W. I. F. David, Chem. Br. 30, 727 (1994).

${ }^{8}$ C. Ambrosch-Draxl, E. Y. Sherman, H. Auer, and T. Thonhauser, Phys. Status Solidi B 241, 1199 (2004).

${ }^{9}$ W. R. Flavell and R. G. Egdell, Chem. Br. 30, 735 (1994).

${ }^{10}$ P. Humbert, Solid State Commun. 60, 21 (1986).

${ }^{11}$ M. Scrocco, J. Electron Spectrosc. Relat. Phenom. 53, 225 (1991).

${ }^{12}$ A. F. Wells, Structural Inorganic Chemistry (Clarendon Press, Oxford, 1984).

${ }^{13}$ F. Quarto, C. Sunseri, S. Piazza, and M. Romano, J. Phys. Chem. 101, 2519 (1997).

${ }^{14}$ Y. Xu and M. A. A. Schoonen, Am. Mineral. 85, 543 (2000).

${ }^{15}$ J. D. Dunitz and L. E. Orgel, Adv. Inorg. Chem. Radiochem. 2, 1 (1960).

${ }^{16}$ J. K. Burdett, Chemical Bonds: A Dialog (Wiley, Chichester 1997).

${ }^{17}$ S. Süzer, S. T. Lee, and D. A. Shirley, Phys. Rev. A 13, 1842 (1976).

${ }^{18}$ I. P. Grant, Adv. Phys. 19, 747 (1970).

${ }^{19}$ P. A. Glans, T. Learmonth, C. McGuinness, K. E. Smith, J. Guo, A. Walsh, G. W. Watson, and R. G. Egdell, Chem. Phys. Lett. 399, 98 (2004).

${ }^{20}$ C. McGuinness, C. B. Stagarescu, P. J. Ryan, J. E. Downes, D. Fu, K. E. Smith, and R. G. Egdell, Phys. Rev. B 68165104 (2003).

${ }^{21}$ W. L. Holstein, J. Phys. Chem. 97, 4224 (1993).

${ }^{22}$ H. P. Geserich, Phys. Status Solidi 25, 741 (1968).

${ }^{23}$ A. W. Sleight, J. L. Gillson, and B. L. Chamberland, Mater. Res. Bull. 5, 807 (1970).

${ }^{24}$ V. N. Shukla and G. P. Wirtz, J. Am. Ceram. Soc. 60, 253 (1977).

${ }^{25}$ V. N. Shukla and G. P. Wirtz, J. Am. Ceram. Soc. 60, 259 (1977).

${ }^{26}$ G. P. Wirtz, C. J. Yu, and R. W. Doser, J. Am. Ceram. Soc. 64, 269 (1981)

${ }^{27}$ A. Goto, H. Yasuoka, A. Hayashi, and Y. Ueda, J. Phys. Soc. Jpn. 61, 1178 (1992).

${ }^{28}$ M. Heinemann, H. J. Terpstram, C. Haas, and R. A. deGroot, Phys. Rev. B 52, 11740 (1995).
${ }^{29}$ I. Hamberg, C. G. Granqvist, K. F. Berggren, B. E. Sernelius, and L. Engstrom, Phys. Rev. B 30, 3240 (1984).

${ }^{30}$ R. L. Weiher and R. P. Ley, J. Appl. Phys. 37, 299 (1966).

${ }^{31}$ J. E. Jaffe, R. Pandey, and A. B. Kunz, Phys. Rev. B 43, 14030 (1991).

${ }^{32}$ Y. Dou, R. G. Egdell, D. S. L. Law, N. M. Harrison, and B. G. Searle, J. Phys.: Condens. Matter 108447 (1998).

${ }^{33}$ P. A. Cox, W. R. Flavell, and R. G. Egdell, J. Solid State Chem. 68, 340 (1987).

${ }^{34}$ J. Nordgren and R. Nyholm, Nucl. Instrum. Methods Phys. Res. A 246, 242 (1986).

${ }^{35}$ J. Nordgren, G. Bray, S. Cramm, R. Nyholm, J. E. Rubensson, and N. Wassdahl, Rev. Sci. Instrum. 60, 1690 (1989).

${ }^{36}$ G. Kresse and J. Hafner, Phys. Rev. B 49, 14251 (1994).

${ }^{37}$ G. Kresse and J. Furthmuller, Comput. Mater. Sci. 6, 15 (1996).

${ }^{38}$ J. P. Perdew, K. Burke, and M. Ernzerhof, Phys. Rev. Lett. 77, 3865 (1996).

${ }^{39}$ P. E. Blöchl, Phys. Rev. B 50, 17953 (1994).

${ }^{40}$ U. Gelius, in Electron Spectroscopy, edited by D. Shirley (North Holland, Amsterdam, 1972).

${ }^{41}$ J. J. Yeh and I. Lindau, At. Data Nucl. Data Tables 32, 1 (1985).

${ }^{42}$ I. M. Band, Y. I. Kharitonov, and M. B. Trzhaskovskaya, At. Data Nucl. Data Tables 23, 443 (1979).

${ }^{43}$ R. G. Egdell, J. Rebane, T. J. Walker, and D. S. L. Law, Phys. Rev. B 59, 1792 (1999).

${ }^{44}$ R. G. Egdell, T. J. Walker, and G. Beamson, J. Electron Spectrosc. Relat. Phenom. 128, 59 (2003).

${ }^{45}$ V. Christou, M. Etchells, O. Renault, P. J. Dobson, O. V. Salata, G. Beamson, and R. G. Egdell, J. Appl. Phys. 88, 5180 (2000).

${ }^{46} \mathrm{P}$. Steiner, H. Höchst, and S. Hüfner, in Photoemission in Solids II, edited by L. Ley and M. Cardona (Springer-Verlag, Berlin, 1979).

${ }^{47}$ D. C. Langreth, Nobel Symp. 24, 210 (1973).

${ }^{48}$ M. Campagna, G. K. Wertheim, H. R. Shanks, F. Zumsteg, and E. Banks, Phys. Rev. Lett. 34, 738 (1975).

${ }^{49}$ J. N. Chazalviel, M. Campagna, G. K. Wertheim, and H. R. Shanks, Phys. Rev. B 16, 697 (1977).

${ }^{50}$ G. K. Wertheim, Chem. Phys. Lett. 65, 377 (1979).

${ }^{51}$ R. G. Egdell, H. Innes, and M. D. Hill, Surf. Sci. 149, 33 (1985).

${ }^{52}$ P. P. Edwards, R. G. Egdell, I. Fragala, J. B. Goodenough, M. R. Harrison, A. F. Orchard, and G. Scott, J. Solid State Chem. 54, 127 (1984).

${ }^{53}$ N. Beatham, P. A. Cox, R. G. Egdell, and A. F. Orchard, Chem. Phys. Lett. 69, 475 (1980).

${ }^{54}$ P. A. Cox, J. B. Goodenough, P. Tavener, D. Telles, and R. G. Egdell, J. Solid State Chem. 62, 36 (1986).

${ }^{55}$ P. A. Cox, R. G. Egdell, J. B. Goodenough, A. Hamnett, and C. C. Naish, J. Phys. C 16, 6221 (1983).

${ }^{56}$ A. Kotani and Y. Toyazawa, J. Phys. Soc. Jpn. 37, 912 (1974).

${ }^{57}$ G. W. Watson and S. C. Parker, J. Phys. Chem. B 10, 1258 (1999).

${ }^{58}$ A. Walsh and G. W. Watson, J. Solid State Chem. (to be published). 